\title{
Stable iPEPO Tensor-Network Algorithm for Dynamics of Two-Dimensional Open Quantum Lattice Models
}

\author{
C. Mc Keever® and M. H. Szymańska๑ \\ Department of Physics and Astronomy, University College London, \\ Gower Street, London, WCIE 6BT, United Kingdom
}

(Received 5 October 2020; revised 3 March 2021; accepted 4 March 2021; published 14 May 2021)

Being able to accurately describe the dynamics steady states of driven and/or dissipative but quantum correlated lattice models is of fundamental importance in many areas of science: from quantum information to biology. An efficient numerical simulation of large open systems in two spatial dimensions is a challenge. In this work, we develop a tensor network method, based on an infinite projected entangled pair operator ansatz, applicable directly in the thermodynamic limit. We incorporate techniques of finding optimal truncations of enlarged network bonds by optimizing an objective function appropriate for open systems. Comparisons with numerically exact calculations, both for the dynamics and the steady state, demonstrate the power of the method. In particular, we consider dissipative transverse quantum Ising, driven-dissipative hard-core boson, and dissipative anisotropic $X Y$ models in non-mean-field limits, proving able to capture substantial entanglement in the presence of dissipation. Our method enables us to study regimes that are accessible to current experiments but lie well beyond the applicability of existing techniques.

DOI: 10.1103/PhysRevX.11.021035

Subject Areas: Atomic and Molecular Physics,

Condensed Matter Physics,

Quantum Physics

\section{INTRODUCTION}

In recent experiments across a variety of architectures, the ability to sustain quantum correlations in a dissipative environment and study the evolution of strongly interacting many-body lattice systems in a precisely controlled manner has progressed enormously. Among these experimental platforms are cavity [1,2] and circuit [3-6] QED systems, arrays of coupled optical cavities [7-9] or of quantum dots [10], hybrid systems [11], polariton lattices [12-19], and certain implementations of ultracold atoms [20].

In the modeling of these systems, the inclusion of degrees of freedom that are external to the lattice, such as a driving field or a bath of oscillators, requires extending the description from a closed to an open quantum lattice model, as illustrated in Fig. 1. Open quantum systems are often well described by a Lindblad master equation [21], which facilitates the study of a range of collective phenomena including nonequilibrium criticality [22-27], quantum chaos [28,29], and time crystallinity [30-32], many of which have no counterparts in closed systems at equilibrium.

Published by the American Physical Society under the terms of the Creative Commons Attribution 4.0 International license. Further distribution of this work must maintain attribution to the author(s) and the published article's title, journal citation, and DOI.
Recent years have seen great strides in the theoretical modeling of open quantum systems [33]; however, to better understand, control, and utilize the dissipative nonequilibrium dynamics of correlated quantum systems, simulation techniques that are scalable to large lattices are still missing, especially in higher dimensions.

The investigation of large many-body quantum systems is hindered by the exponential growth of the Hilbert space. As the size of the system increases, solving the Lindblad master equation exactly using methods such as

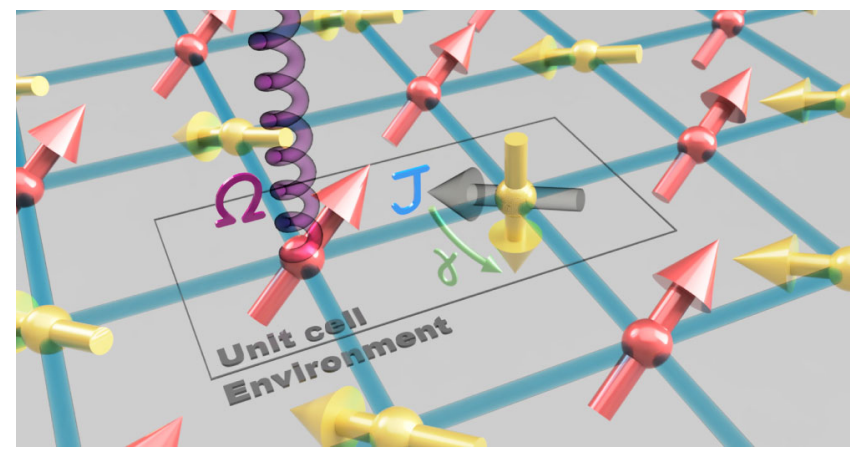

FIG. 1. Open quantum lattice model of interacting spins. Nearest-neighbor spins are coupled via a hopping $J$ and interact with an external bath via a coherent drive $\Omega$ and/or a dissipative process $\gamma$. The open system can be modeled by describing the unit cell and its environment using a tensor network. 
diagonalization of the Liouvillian or averaging over ensembles of exact quantum trajectories [34-36] quickly become infeasible. To simplify the problem, many have resorted to a mean-field-type approximation [25,37-43] in which correlations between small individual subsystems are approximated by an average field. However, this simplification may often give qualitatively incorrect results in regions where intersubsystem correlations become important-for instance, near criticality. Moreover, key aspects such as entanglement and quantum information cannot be treated at this level. Progressing beyond meanfield approximations should therefore involve the systematic inclusion of correlations between subsystems in a controlled and tractable manner.

In this vein, phase-space methods such as those based on the Wigner [44], positive-P [45], and Q [46] representations attempt to find classical stochastic processes for which the hierarchy of coupled moments is a good approximation to that of the quantum problem. For highly nonlinear problems, phase-space techniques often fail dramatically in important regimes [47-49]. Cluster-based methods [24,50] separate large lattices into small clusters and capture correlations within lattice sites belonging to each cluster, an approach that can become inaccurate when correlation lengths exceed cluster sizes. Variational approaches $[51,52]$ based on the parametrization of the state in terms of a suitable functional and their optimization rely on good intuition, which may not be available for some problems. Recently, methods based on neural networks and the variational minimization of an appropriate cost function [53-55] have provided an interesting proof of concept; however, like most of these methods, they are restricted to small system sizes or may fail to capture long-range correlations.

A different approach is to restrict the growth of the system's Hilbert space by retaining only the most important correlations or most probable states [56]. Tensor network (TN) methods [57] belong to this class. Here, truncation of Hilbert space is controlled by the so-called bond dimension (usually denoted $D$ or $\chi$ ) of indices that connect a set of tensors representing the quantum state. In the context of closed quantum many-body systems, the significant success of TN methods is underpinned by an area law in the growth of entanglement entropy possessed by ground states of gapped Hamiltonians [58]. For open systems, the picture is less clear. In particular, it is not obvious whether transient or steady states can be efficiently represented by a TN. Nevertheless, in the context of dissipative or drivendissipative systems, we can reasonably expect that in many cases, dissipative processes should curtail the growth of entanglement and limit correlations generated by entangling dynamics; this is found to hold true in the fixed points of rapidly mixing dissipative quantum systems, which obey an area law [59-61].

Despite this expectation, $\mathrm{TN}$ algorithms for open systems [62-66] have mostly been restricted to one-dimensional lattices, where the simple geometry plays a central role in the algorithm. In dimensions greater than one, progress has been limited. The work of Ref. [67] introduced the infinite projected entangled pair operator (iPEPO) to represent the mixed state of an infinite-periodic two-dimensional square lattice and employed the so-called simple update (SU) algorithm to apply the Lindblad dynamical map, evolving the system in real time towards a steady state. Although SU is efficient, in order to integrate the equation of motion, it isolates a subsystem-for example, one unit cell-from the rest of the lattice and applies the dynamical map to the subsystem in isolation until a steady state is reached. It has been questioned whether this approach can produce accurate results, and there are concerns over the convergence of this method in non-mean-field regimes [68]. While algorithms going beyond SU exist for closed and finite temperature systems [69-71], advancing beyond the SU approach in the driven-dissipative context remains undeveloped.

In this paper, we devise a new TN method to accurately simulate time dynamics and steady states of many-body quantum lattice models in two spatial dimensions and directly in the thermodynamic limit. The method uses the iPEPO as an ansatz for the mixed state of the open system and incorporates techniques inspired by those presented in Ref. [72] - full environment truncation (FET) and fixing the network to weighted trace gauge (WTG) - to calculate accurate time dynamics and steady-state solutions of open quantum lattice models. The central step in the algorithm involves finding an optimal truncation of enlarged bonds with respect to an objective function appropriate for mixed quantum states.

The method successfully reproduces numerically exact calculations for both dynamics and steady states while also agreeing with results obtained using the so-called cornerspace renormalization method of Ref. [56]. Importantly, it performs well in non-mean-field limits, proving able to capture substantial correlations in the presence of dissipation and therefore enabling the study of regimes that are accessible to current experiments but lie well beyond the applicability of existing techniques.

The paper is organized as follows. In Sec. II, we describe the algorithm, including a brief introduction to the Lindblad master equation and the TN ansatz. As a benchmark, we calculate the time dynamics of a dissipative transverse quantum Ising model in Sec. III A and find that the systematic inclusion of correlations - controlled by the TN bond dimension-coupled with the incorporation of the unit cell's environment when truncating enlarged bonds, yields results that agree very well with the exact dynamics. Furthermore, we demonstrate the applicability of the algorithm outside the exactly solvable regime. In Sec. III B, we show that the FET method outperforms the SU method by finding more optimal truncations of enlarged bonds and removing redundant internal correlations in the 
network. In Sec. III C, we show that lattice models with drive and dissipation can also be treated using this method, and we compare steady-state results for a driven-dissipative hard-core boson model with the values in the literature. In Sec. III D, we apply the algorithm to a dissipative anisotropic $X Y$ model and show that the iPEPO steadystate solution is qualitatively different from the meanfield theory. Finally, in Sec. IV, we conclude with a short discussion.

\section{ALGORITHM}

\section{A. Master equation}

The goal of the algorithm is to calculate time dynamics and steady states of translationally invariant, twodimensional, quantum lattice models, which interact with a bath via a Lindblad master equation $(1)(\hbar=1)$,

$$
\frac{d \hat{\rho}}{d t}=\hat{\mathcal{L}}(\hat{\rho})=-i[\hat{\mathcal{H}}, \hat{\rho}]+\hat{\mathcal{D}}(\hat{\rho})
$$

where $\hat{\mathcal{H}}$ governs the coherent dynamics of the system and the dissipator $\hat{\mathcal{D}}$, which models the coupling of the system to its bath, has the form

$$
\hat{\mathcal{D}}(\hat{\rho})=\sum_{\alpha}\left(\hat{L}_{\alpha} \hat{\rho} \hat{L}_{\alpha}^{\dagger}-\frac{1}{2}\left\{\hat{L}_{\alpha}^{\dagger} \hat{L}_{\alpha}, \hat{\rho}\right\}\right),
$$

with $\hat{L}_{\alpha}$ being the Lindblad operators. We focus on the case of time-independent nearest-neighbor Hamiltonians such that $H$ can be decomposed as a sum of Hermitian operators that act nontrivially on at most two nearest-neighbor lattice sites. Although the algorithm allows for up to two local dissipators, for simplicity, we focus only on local coupling to the environment such that each Lindblad operator acts on one site only and respects the translational invariance of the Hamiltonian.

\section{B. TN ansatz}

We represent the system's density matrix $\rho(t)$ as an iPEPO. The iPEPO is composed of a network of tensors $\left\{A_{j}\right\}$, where we associate each node $j$ of the network with one site of the square lattice shown in Fig. 2(a). To reflect the translational invariance of the system and to simplify the algorithm, we use a pair of independent tensors $A_{j}$ and $A_{l}$ to represent the unit cell. The infinite system is the repetition of this unit cell over the two-dimensional plane. Each sixth-rank tensor $A$ has a pair of physical indices of dimension $d$ and a set of four bond indices of dimension $D$, reflecting the coordination number $z=4$ associated with a square lattice. The physical dimension $d$ corresponds to the dimension of the local Hilbert space at each lattice site ( $d=2$ for the two-level spin), whereas $D$ is a variational parameter that controls the accuracy of the ansatz. It is convenient to use the vectorized form of the density (a)

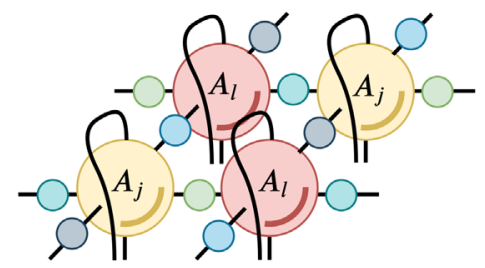

(b)

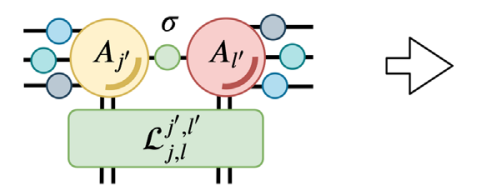

(c) (e)

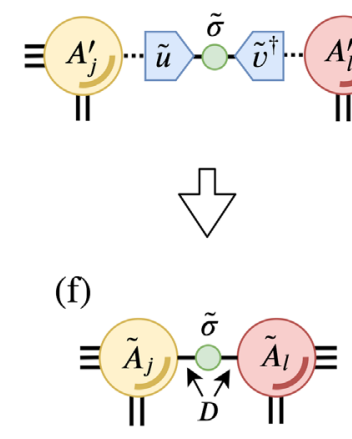

(d)
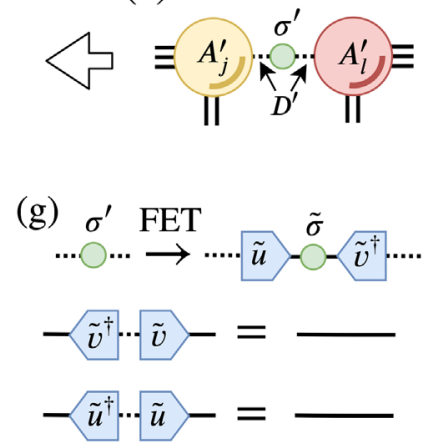

FIG. 2. Main steps in the time evolution algorithm. (a) Vectorized form of the iPEPO. (b) Contraction of $A_{j^{\prime}}$ and $A_{l^{\prime}}$ with the dynamical map $\mathcal{L}$. (c) SVD of $e^{\tau \mathcal{L}} A_{j} A_{l}$. (d) $D^{\prime}$ singular values of relative tolerance greater than $\epsilon_{D^{\prime}}$ retained in the diagonal bond matrix $\sigma^{\prime}$. (e) Isometries $\tilde{u}$ and $\tilde{v}$, which truncate the enlarged bond from $D^{\prime}$ to $D$, giving the new bond matrix $D$. (f) Updated tensors $\tilde{A}_{j}$ and $\tilde{A}_{l}$. (g) Full environment truncation algorithm to find the isometries $\tilde{u}$ and $\tilde{v}$, which maximize the fidelity between truncated and untruncated bonds.

operator, which, at the level of the iPEPO, corresponds to vectorization of the pair of local Hilbert space indices as shown in Fig. 2(a) and has the effect of transforming the iPEPO into the form of a infinite projected entangled pair state (iPEPS) commonly used in TN algorithms for twodimensional closed systems [57]. Finally, to each unique bond, we associate a bond matrix $\sigma$.

As with other algorithms based on matrix product operators (MPOs), the PEPO ansatz is not inherently positive, and therefore not all PEPOs represent physical states. For the present case of an iPEPO, we do not have access to the full spectrum of eigenvalues, and it has been shown for the case of MPOs that the problem of deciding whether a given iMPO represents a physical state in the thermodynamic limit is provably undecidable [73]. Therefore, we rely on the positivity of the dynamical map to maintain the physicality of the iPEPO throughout the time evolution and find in practice that, in most 

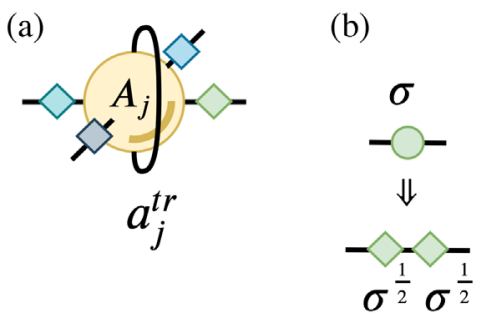

(d)

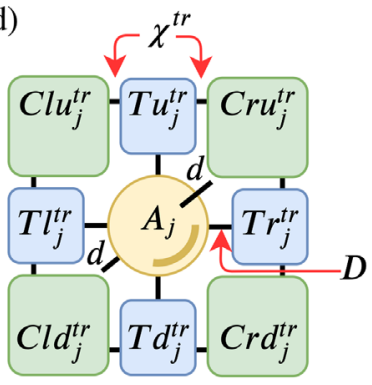

$\rho_{j}=\mathcal{E}_{j}^{t r} A_{j}$ (c)

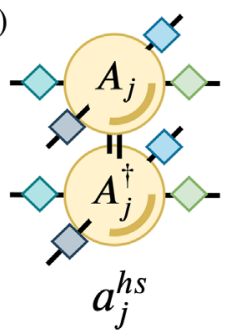

truncation of enlarged bond dimensions as discussed in Sec. II D. In both cases, we calculate the effective environment using a corner transfer matrix method [74-79]. In particular, we use a variant of the corner transfer matrix renormalization group (CTMRG) algorithm [80] that makes use of an intermediate SVD to improve the stability, details of which are given in the Appendix A.

\section{Time evolution}

To calculate dynamics and find a $\mathrm{TN}$ representation of the steady state, we use a time-evolving block decimation (TEBD) algorithm. The time evolution is obtained by application of the dynamical map $\rho_{t}=e^{t \mathcal{L}} \rho_{0}$. In principle, it may also be possible to find the steady state directly by searching for the ground state of the Hermitian operator $\rightarrow \mathcal{L}^{\dagger} \mathcal{L}$, for example, via imaginary time evolution. However, in general, $\mathcal{L}^{\dagger} \mathcal{L}$ is a highly nonlocal operator; therefore, it is not straightforward to implement it using standard techniques for an infinite system [81]. Finally, access to the transient dynamics is often of direct interest in many physical contexts.

The dynamical map $e^{t \mathcal{L}}$ is approximated by a set of Trotter layers as is common in algorithms based on TEBD. In particular, consider the evolution of the state from a time $t$ to a short time later $t+\tau$; then, in vectorized notation, where we note that the density matrix is vectorized column by column, the dynamical map takes the form

$$
\rho(t+\tau)=e^{\tau \mathcal{L}} \rho(t) .
$$

cases, the reduced density matrices calculated from the iPEPO are physical.

We refer to all of the spins in the system that are not part of the unit cell as its environment (see Fig. 1.), not to be confused with the system's bath, which is accounted for in the Lindblad master equation (1). Since the system is infinite, we represent the environment approximately by associating to each tensor in the unit cell an effective environment $\mathcal{E}$. Note that $\mathcal{E}$ is, itself, made up of a set of tensors including four corner transfer matrices $C_{\mu \nu}$ and four half-row or half-column tensors $T_{\mu}$, where the labels $\mu$ and $\nu$ take the appropriate first letter of left, right, up, and down as illustrated in Figs. 3(d) and 3(e).

We consider two distinct types of effective environments. The trace effective environment $\mathcal{E}^{\text {tr }}$ of Fig. 3(d) is calculated by first tracing over the local Hilbert space dimensions $d$ of the tensors at each node of the network, giving the set of fourth-rank tensors $\left\{a_{j}^{\text {tr }}\right\}$ as shown in Fig. 3(a). We use $\mathcal{E}^{\text {tr }}$ to calculate the reduced density matrices of the system. Second, the Hilbert-Schmidt effective environment $\mathcal{E}^{\mathrm{HS}}$ [Fig. 3(e)] is that formed by first contracting $a_{j}^{\mathrm{HS}}=\operatorname{tr} A_{j} A_{j}^{\dagger}$, giving the Hilbert-Schmidt inner product, where all bond indices $\{D\}$ are left open as shown in Fig. 3(c). Note that $\mathcal{E}^{\mathrm{HS}}$ is used during the algorithm to calculate an optimal

The Liouvillian superoperator $\mathcal{L}$ is two-local and can therefore be written as a sum of superoperators acting on nearest neighbors of the square lattice, where the labels $\alpha$ and $\beta$ correspond to the coordinates of the lattice sites $j$ and $l$, respectively. The full Liouvillian takes the form

$$
\mathcal{L}=\sum_{\langle\alpha, \beta\rangle} \mathcal{L}_{\alpha, \beta}=\sum_{\langle\alpha, \beta\rangle} \mathcal{H}_{\alpha, \beta}+\mathcal{D}_{\alpha, \beta}
$$

The Hamiltonian part of the evolution is included in the superoperator $\mathcal{H}$ and the dissipative part in the superoperator $\mathcal{D}$; each is constructed as shown in Eqs. (5) and (6), respectively:

$$
\begin{gathered}
\mathcal{H}_{\alpha, \beta}=-i\left(\mathbb{I}_{\alpha, \beta} \otimes H_{\alpha, \beta}-H_{\alpha, \beta}^{T} \otimes \mathbb{I}_{\alpha, \beta}\right), \\
\mathcal{D}_{\alpha, \beta}=\frac{1}{2}\left(2 L_{\alpha, \beta}^{*} \otimes L_{\alpha, \beta}-I_{\alpha, \beta} \otimes L^{\dagger} L_{\alpha, \beta}-L^{T} L_{\alpha, \beta}^{*} \otimes I_{\alpha, \beta}\right) .
\end{gathered}
$$

We then split the vectorized operators in the exponent into those acting on even and odd pairs of lattice sites along both the $x$ and $y$ lattice dimensions, giving four sets of vectorized operators $\mathcal{L}_{x}^{e}, \mathcal{L}_{x}^{o}, \mathcal{L}_{y}^{e}$, and $\mathcal{L}_{y}^{o}$, where 


$$
\mathcal{L}_{r}^{e}=\sum \mathcal{L}_{2 r, 2 r+1}, \quad \mathcal{L}_{r}^{o}=\sum \mathcal{L}_{2 r-1,2 r}
$$

which allows us to decompose $e^{t \mathcal{L}}$ into a set of layers via a Trotter decomposition with $\tau=t / n$, where $n \gg 1$ is the Trotter number with

$$
e^{\tau \mathcal{L}}=e^{\tau \mathcal{L}_{x}^{e}} e^{\tau \mathcal{L}_{x}^{o}} e^{\tau \mathcal{L}_{y}^{e}} e^{\tau \mathcal{L}_{y}^{o}}+\mathcal{O}\left(\tau^{2}\right) .
$$

Each dynamical map in the decomposition is applied to pairs of nearest-neighbor tensors $A_{j}$ and $A_{l}$ in turn. We first construct the linear map $\mathcal{L}\left(A_{j} A_{l}\right)$, where the linear operator $\mathcal{L}_{j, l}^{j^{\prime}, l^{\prime}}$ acts on the pair of tensors $A_{j}$ and $A_{l}$ such that $A_{j} A_{l}$ behaves as a vector in the linear map, as illustrated in Fig. 2(b). By repeated application of this map, an approximation to the tensor $e^{\tau \mathcal{L}} A_{j} A_{l}$ [Fig. 2(c)] is calculated using Krylov subspace methods, eliminating the need for explicit calculation of $e^{\tau \mathcal{L}}$, where $\tau$ is a real number for the case of real time evolution.

To complete the update, the resulting tensor $A_{j, l}^{\prime}=$ $e^{\tau \mathcal{L}} A_{j} A_{l}$ needs to be decomposed into a new pair of tensors $A_{j}^{\prime}$ and $A_{l}^{\prime}$, illustrated in Figs. 2(c) and 2(d). Typically, this decomposition is accomplished via singular value decomposition (SVD), where, in general, the new bond dimension $D^{\prime}$-equal to the number of singular values associated with the SVD-will be enlarged $\left(D^{\prime}>D\right)$ and therefore needs to be truncated in an appropriate way for the algorithm to remain efficient; in particular, we would like to truncate $D^{\prime}$ back to $D$ after each dynamical map.

\section{Truncation of enlarged bonds}

For TNs without closed loops (acyclic), finding an optimal truncation benefits greatly from the ability to efficiently apply a gauge transformation and recast a network to a so-called canonical form; for details, we refer the reader to Ref. [57]. For TNs with closed loops (cyclic), however, such a canonical form cannot be defined uniquely, and truncating the enlarged bond in an optimal way is much less straightforward. Moreover, cyclic TNs can host so-called internal correlations, which have no influence on the properties of the quantum state but can cause computational problems if they are allowed to accumulate [72].

After applying the dynamical map, we choose to decompose the tensors using SVD and truncate the bond irrespective of the state of the environment, leaving a new dimension $D^{\prime} \geq D$ chosen such that only those singular values greater than some small tolerance $\epsilon_{D^{\prime}} \ll 1$ are retained. We are then left with a bond matrix $\sigma$ with the remaining $D^{\prime}$ singular values along its diagonal and the tensors $A_{i}$ and $A_{j}$ as shown in Fig. 2(d). The final step in the truncation involves replacing $\sigma$ with the product $\tilde{u} \tilde{\sigma} \tilde{v}^{\dagger}$, where $\tilde{u}$ and $\tilde{v}$ are isometries of dimension
$\left(D^{\prime}, D\right)$ such that $\tilde{u} \tilde{u}^{\dagger}=\tilde{v} \tilde{v}^{\dagger}=I$ and $\tilde{\sigma}$ is a new $D$-dimensional diagonal bond matrix. The enlarged bond is then truncated by contracting $A_{j}$ and $A_{l}$ with $\tilde{u}$ and $\tilde{v}$ as illustrated in Fig. 2(e).

To calculate the set $\tilde{u}, \tilde{\sigma}$, and $\tilde{v}$, we adapt the FET algorithm of Ref. [72], which prescribes a method to find the truncation of an internal index of an arbitrary network for closed systems, which is optimal with respect to a fidelity measure for pure states. In our case, since we are dealing with an open system, we optimize the truncation with respect to an objective function suitable for mixed states. More precisely, we maximize a mixed-state fidelity measure between the state $\rho$ in which the enlarged bond dimension is left untruncated and the state $\phi$ in which the same bond has been truncated by $\tilde{u}, \tilde{\sigma}$, and $\tilde{v}$. Supposing that a global maximum is found, this procedure finds the isometries that leave $\phi$ as close as possible to $\rho$ with respect to the chosen fidelity measure.

We choose to maximize the fidelity $\mathcal{F}(\rho, \phi)$, which has the Hilbert-Schmidt inner product of $\rho$ and $\phi$ in its numerator and the geometric mean of their purities $\operatorname{tr}\left(\rho^{2}\right)$ and $\operatorname{tr}\left(\phi^{2}\right)$ in its denominator [82],

$$
\mathcal{F}(\rho, \phi)=\frac{\operatorname{tr}(\rho \phi)}{\sqrt{\operatorname{tr}\left(\rho^{2}\right) \operatorname{tr}\left(\phi^{2}\right)}} .
$$

Since squaring $\mathcal{F}$ is convex, the $\rho$ and $\phi$ that maximize $\mathcal{F}^{2}(\rho, \phi)$ also maximize $\mathcal{F}(\rho, \phi)$. We therefore construct $\mathcal{F}^{2}(\rho, \phi) \operatorname{tr}\left(\rho^{2}\right)$ as a Rayleigh quotient of tensors, which can be maximized to find an optimal $\tilde{u}, \tilde{\sigma}$, and $\tilde{v}$. Details of the optimization procedure are given in Appendix B.

Finally, there exists a gauge freedom across the newly truncated bond, which we fix to so-called weighted trace gauge (WTG) as described in Ref. [72]. This gauge freedom allows for the recycling of the environment $\mathcal{E}^{\mathrm{HS}}$ calculated for use at each FET step of the algorithm as an initial guess for the renormalization procedure (CTMRG, in our case), which precedes the following FET step, thereby reducing the number of renormalization iterations required at each step. We refer to the algorithm outlined in this section as full environment truncation in weighted trace gauge (FET + WTG).

It is straightforward to recover a SU method by bypassing the FET and WTG steps above and instead choosing both $\tilde{u} \rightarrow \tilde{u}_{\mathrm{SU}}$ and $\tilde{v} \rightarrow \tilde{v}_{\mathrm{SU}}$ as $D^{\prime} \times D$ matrices with all diagonal entries equal to one and all other entries equal to zero and by retaining the $D$ largest singular values of $\sigma^{\prime}$ in the truncated $\tilde{\sigma}_{\mathrm{SU}}$. In general, the set of $\tilde{u}, \tilde{v}$, and $\tilde{\sigma}$ that we find using FET is not equivalent to the set $\tilde{u}_{\mathrm{SU}}, \tilde{v}_{\mathrm{SU}}$, and $\tilde{\sigma}_{\mathrm{SU}}$, showing that, in the general case, SU does not yield a truncation that is optimal with respect to the objective function we use. A comparison between SU and FET + WTG is made in Sec. III B. 


\section{RESULTS}

\section{A. Dissipative transverse Ising model}

As a first benchmark of the algorithm, we simulate the dynamics of a dissipative transverse quantum Ising model with Hamiltonian

$$
\hat{H}=\frac{V}{z} \sum_{\langle j, l\rangle} \hat{\sigma}_{j}^{z} \hat{\sigma}_{l}^{z}+\sum_{j} \frac{h_{x}}{2} \hat{\sigma}_{j}^{x}
$$

where $V$ is the hopping coupling, $h_{x}$ is the strength of a transverse field, and $z$ is the lattice coordination number, which we set to $z=4$ for the square lattice. The spins undergo dissipation at a rate $\gamma$ described by local Lindblad jump operators $\hat{L}_{j}=\sqrt{\gamma} \frac{1}{2}\left(\hat{\sigma}_{j}^{y}-i \hat{\sigma}_{j}^{z}\right)$, which are the same at each lattice site. For zero transverse field $h^{x} / \gamma=0$, the purely dissipative dynamics $\mathcal{D}\left(\rho_{\text {dis }}\right)=0$ drive the system towards a steady state $\rho_{\text {dis }}=\otimes\left|\downarrow_{x}\right\rangle\left\langle\downarrow_{x}\right|$, which does not commute with the Hamiltonian; thus, ordered phases of the Hamiltonian can be frustrated by the dissipation. Moreover, in the specific case of $h^{x} / \gamma=0$, this Liouvillian belongs to a family of efficiently solvable dissipative models [83] (see Appendix D for further details) in which correlations remain localized; therefore, the Liouvillian admits an efficient, exact solution for local observables. We denote this method EXACT and use it as a benchmark.

For all parameters considered, we initialize the lattice spins in a product state $\rho_{0}=\otimes\left|\uparrow_{z}\right\rangle\left\langle\uparrow_{z}\right|$ and simulate their evolution in time in strongly dissipative $(V / \gamma=0.2$, $\left.h^{x} / \gamma=0\right)$, moderately dissipative $\left(V / \gamma=1.2, h^{x} / \gamma=1.0\right)$, and weakly dissipative $\left(V / \gamma=4.0, h^{x} / \gamma=0\right)$ regimes, as well as in a regime $\left(V / \gamma=0.5, h^{x} / \gamma=1.0\right)$, which does not admit an efficient solution using the EXACT method. For all results pertaining to this model, we choose $\epsilon_{D^{\prime}}=10^{-8}$ and set the convergence criteria for both the CTMRG and FET algorithms to $10^{-10}$. We choose a time step $\tau \gamma=0.01$ in all cases except for the weakly dissipative regime, where we choose $\tau \gamma=0.005$.

In each regime, we calculate reduced density matrices $\rho_{j}$ and $\rho_{l}$ for each lattice site labeled $j$ and $l$ in the two-site unit cell, as well as the set of four nearest-neighbor reduced density matrices $\rho_{j l}$ and four next-nearest-neighbor reduced density matrices $\rho_{j j^{\prime}}$, where $j$ and $j^{\prime}$ are at a distance of 2 lattice constants rather than $\sqrt{2}$ (i.e., they are in the same row or column). Although we find that all reduced density matrices within each set are equivalent to a high precision, it is convenient to plot expectation values averaged over each set. We therefore calculate the average magnetization $m^{x}=\frac{1}{2}\left(\operatorname{tr}\left(\hat{\sigma}^{x} \hat{\rho}_{j}\right)+\operatorname{tr}\left(\hat{\sigma}^{x} \hat{\rho}_{l}\right)\right)$ as well as the average purity of the single-site reduced density matrices, $\Pi_{1}=\frac{1}{2}\left(\operatorname{tr}\left(\hat{\rho}_{j}^{2}\right)+\operatorname{tr}\left(\hat{\rho}_{l}^{2}\right)\right)$, as a function of time. To compare larger reduced density matrices, we calculate $S_{12}^{x x}$ and $S_{13}^{x x}$, where $S_{j l}^{x x}(t)=\operatorname{tr}\left(\hat{\sigma}_{j}^{x} \otimes \hat{\sigma}_{l}^{x} \rho^{t}\right)$, again averaged over the four possible choices for $j$ and $l$. Finally, we show the infidelity
$\mathcal{I}(t)=1-\mathcal{F}(t)$ of each truncation averaged over the four Trotter layers that make up every time step $\tau$ where $\mathcal{F}$ is the mixed-state fidelity equation (9). Results are plotted for a range of bond dimensions $D$ and the environment dimensions $\chi^{\mathrm{tr}}$ and $\chi^{\mathrm{HS}}$, where we choose $\chi^{\mathrm{tr}}=\chi^{\mathrm{HS}}=\chi$ in each case, and where $\chi^{\mathrm{tr}}$ and $\chi^{\mathrm{HS}}$ are associated with the effective environments $\mathcal{E}^{\mathrm{tr}}$ and $\mathcal{E}^{\mathrm{HS}}$, respectively. Finally, we have confirmed the convergence of the results with respect to increasing $\chi^{\mathrm{tr}}$ and $\chi^{\mathrm{HS}}$ in all results shown.

\section{Strong dissipation}

In Fig. 4(a), we plot the results of the strongly dissipative regime, in which the dissipative process dominates and where the spins are strongly damped. The exact dynamics of the system can be summarized as follows. From the initial product state, the average single-site expectation value $\operatorname{tr}\left(\sigma^{x} \rho_{t}\right)$ decays monotonically in time towards a steady state, which reflects the strong spin damping. Each spin is initially in a pure state with $\operatorname{tr}\left(\rho_{t}^{2}\right)=1$ and becomes mixed during the dynamics, eventually tending towards a purity of $\operatorname{tr}\left(\rho_{t}^{2}\right) \approx 0.88$ after the transient evolution. From an initially uncorrelated state, spin-spin correlations become nonzero and remain finite after the transient phase.

Comparing the results of FET + WTG with the exact solution, we find that excellent convergence is achieved for $D=4$ and $D=5$, while the results for $D=2$ and $D=3$ fall somewhere between the "mean-field" $D=1$ solution and the exact solution. The $D=1$ solution tends towards an uncorrelated product state of spins in the $\left|\downarrow^{x}\right\rangle$ phase, which again reflects the dominance of the dissipative dynamics in the solution of the mean-field theory. As correlations are included by increasing $D$ to $D=2$ and $D=3$, we find that $S_{12}^{x x}$ and $S_{13}^{x x}$ become nonzero, and for $D=3$, the solution follows the exact dynamics closely at early times; however, after the transient stage, the spins tend towards an almost pure steady state in the $\left|\downarrow^{x}\right\rangle$ phase, similar in character to the $D=1$ solution. Upon increasing to $D=4$ and $D=5$, we see that the FET + WTG method reproduces the exact dynamics to excellent precision across all observables calculated.

Figure 4(a.v) plots the infidelity of truncation $\mathcal{I}(t)$, the qualitative behavior of which is similar for all values of $D$. As the dynamics progresses from the initial product state and correlations begin to deviate from zero, $\mathcal{I}(t)$ increases from $\mathcal{I} \ll 1$, where the error introduced by truncation of enlarged bonds is negligible, to a larger finite value, which indicates that the truncation causes the state to deviate slightly from the exact dynamics; nevertheless, for $D=4$ and $D=5, \mathcal{I}(t)$ remains below approximately $10^{-10}$ at all times and is an indicator of the accuracy of the results. We note here that $\mathcal{I}(t)$ has a dependence on the time step $\tau$, which should be considered when comparing this parameter across different values of $\tau$. 

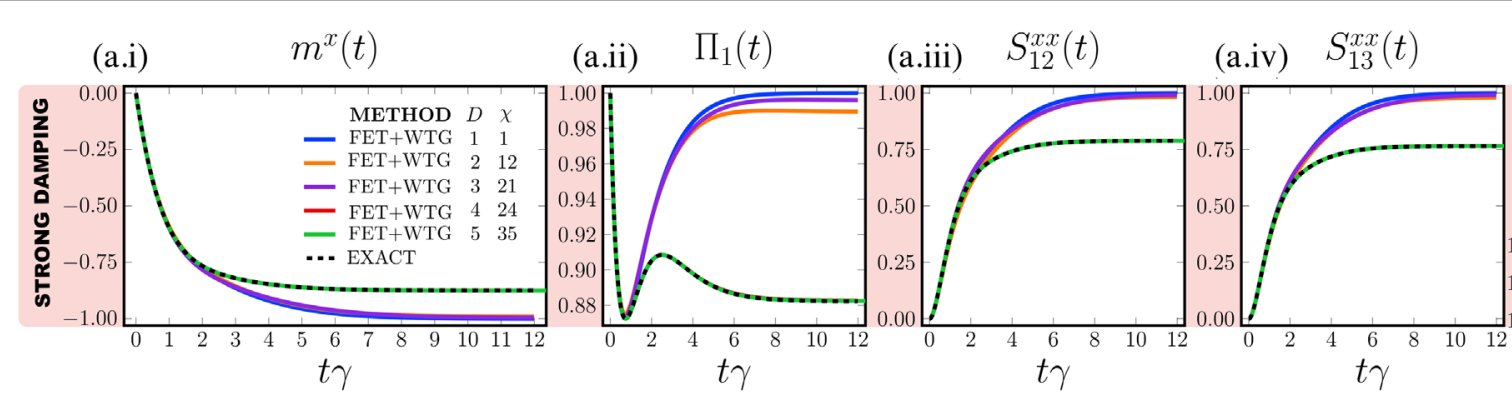

(a.v) $\mathcal{I}(t)$

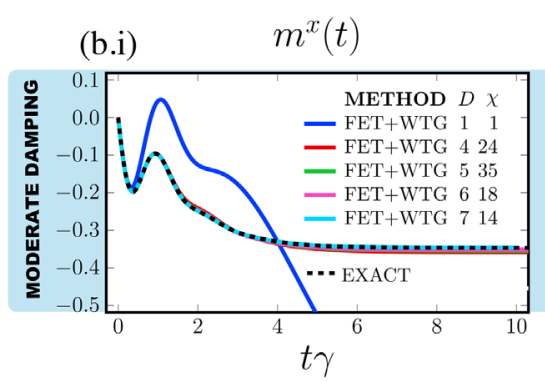

(b.ii) $\Pi_{1}(t)$

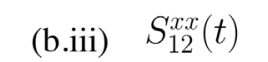

(b.iv) $S_{13}^{x x}(t)$
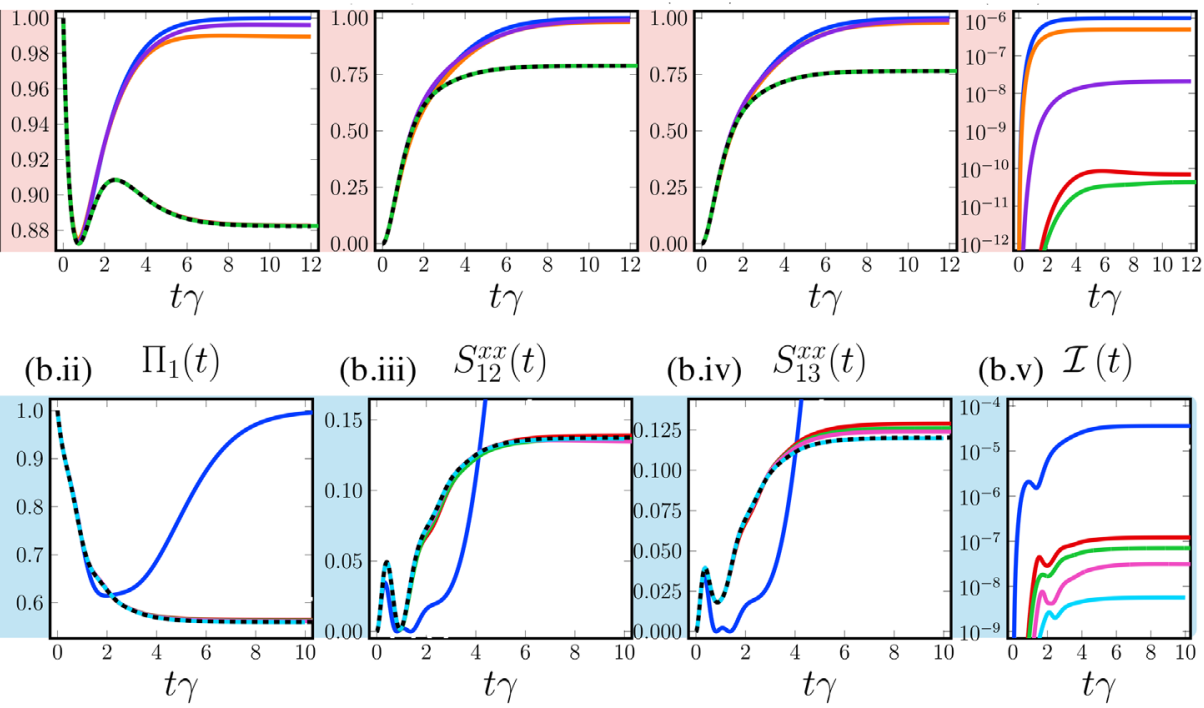

(b.v) $\mathcal{I}(t)$
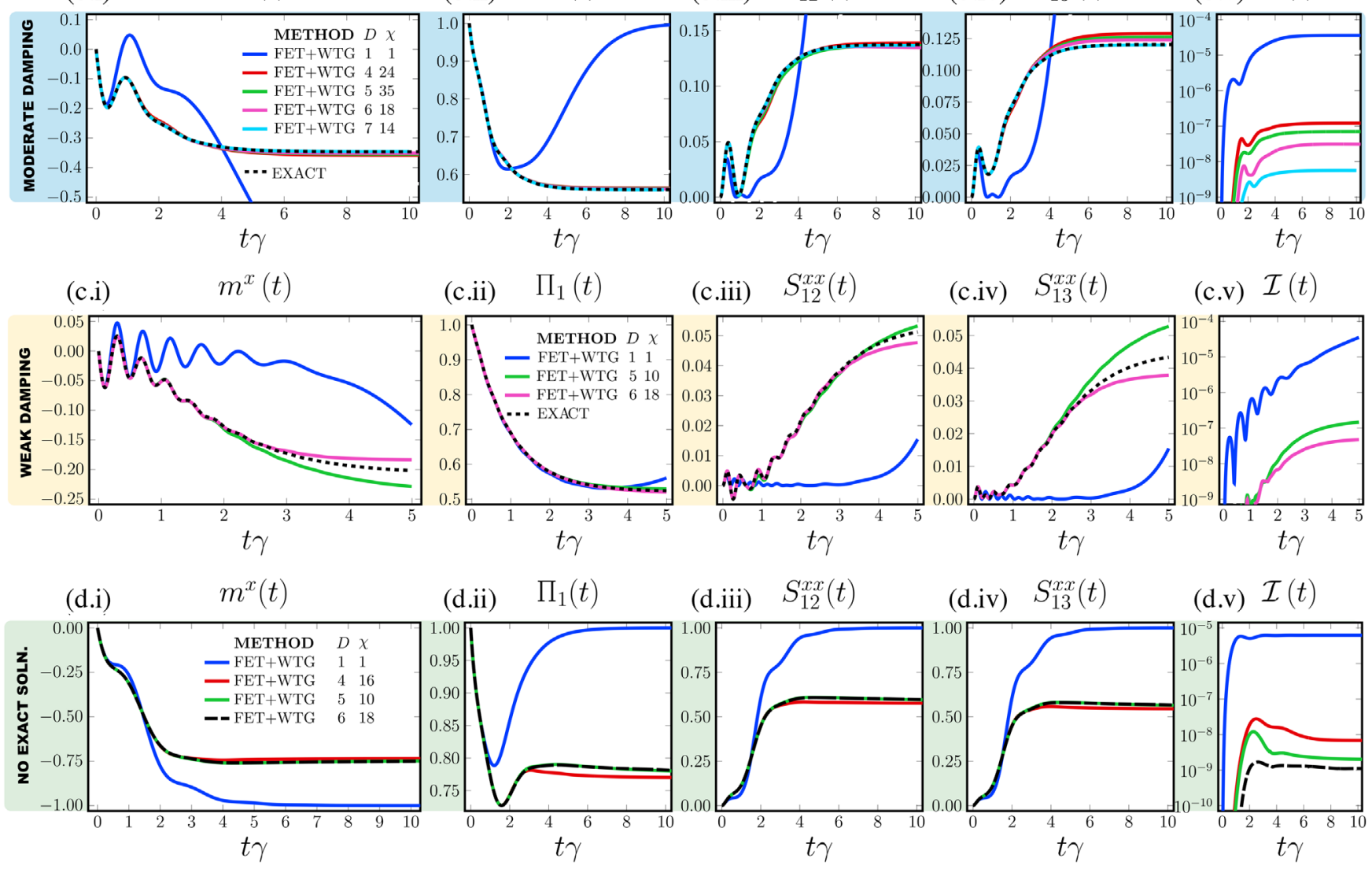

(c.v) $\mathcal{I}(t)$

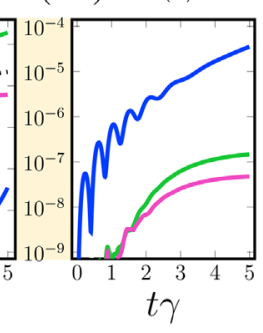

FIG. 4. Dynamics of the dissipative Ising model for $h_{x} / \gamma=0$ in (a) strong $(V / \gamma=0.2)$, (b) moderate $(V / \gamma=1.2)$, and (c) weak $(V / \gamma=4.0)$ spin-damping regimes calculated using FET + WTG for a range of bond dimensions $D$ and superimposed with results calculated using the EXACT method. (d) Results for a regime not applicable to the EXACT method $\left(V / \gamma=0.5\right.$ and $\left.h_{x} / \gamma=1.0\right)$ but that can be treated with FET + WTG. In each case, we plot (i) the magnetization $m^{x}(t)$, (ii) the average purity $\Pi_{1}$ of the single-site reduced density matrices, (iii) the nearest-neighbor $S_{12}^{x x}$, (iv) the next-nearest-neighbor $S_{13}^{x x}$ spin-spin correlations, and (v) the average infidelity of truncation $\mathcal{I}(t)$ at each time step.

\section{Moderate and weak dissipation}

An example of the moderate dissipation regime is presented in Fig. 4(b). In this case, the hopping strength is comparable to the dissipation, and therefore, the exact dynamics display some transient oscillations, which are quickly damped by the dissipation. Here, again, the exact solution differs significantly from the $D=1$ solution in which the dynamics tend towards a pure steady state with all spins in the $|\downarrow\rangle$ state. We find that FET + WTG reproduces the exact dynamics to a good precision for the single-site observables for $D>3$, while $S_{12}^{x x}$ and $S_{13}^{x x}$ also show very good agreement with EXACT, particularly for $D=7$.

A weak dissipation case for $V / \gamma=4.0$ and $h^{x} / \gamma=0.0$ is plotted in Fig. 4(c). The weakly damped oscillations of the EXACT results at early times reflect the dominance of the hopping term in this regime. While the $D=1$ solution gives incorrect results, the results for $D=5$ and $D=6$ reproduce the exact solution early in the transient phase and begin to deviate from the exact dynamics after approximately $t \gamma=$ 2-3 while still retaining the same qualitative behavior. The fact that a larger bond dimension is required to reproduce the 
exact results is indicative of the greater role played by correlations in this coherent hopping-dominated regime.

\section{Outside the exactly solvable regime}

For finite transverse field $h^{x}$, the Lindblad master equation does not fulfill the conditions for an efficient, exact solution using the EXACT method, and correlations may not remain localized; nevertheless, FET + WTG makes no assumption as to the extent of correlations and should therefore be applicable for these parameters. As an example, a case for $V / \gamma=0.5$ and $h^{x} / \gamma=1.0$ is presented in Fig. 4(d). Using FET + WTG, we find that the dynamics converge as the $\mathrm{PEPO}$ bond dimension is increased. Results for $D \in[1,4,5,6]$ converge very well for $D \geq 5$. The behavior of the system is similar to the efficiently solvable cases; after some transient phase, the initial pure product state tends towards a correlated mixed state, which is qualitatively different from the mean-field solution. The infidelity of truncation in Fig. 4(d.v)remains below $\mathcal{I}(t)<10^{-8}$ for the converged results, which is in line with previous benchmarking results.

\section{B. Comparison with simple update}

To highlight differences between the FET + WTG and SU truncation methods, we compare the results calculated using each method in the moderate damping regime $\left(V / \gamma=1.2, h^{x} / \gamma=0\right)$ of Sec. III A for a range of bond dimensions. All parameters are the same for both methods: $\tau=0.01$ and $\epsilon_{D^{\prime}}=10^{-8}$ and CTRMG and FET convergence criteria set to $10^{-10}$, with the only difference being how $\tilde{u}, \tilde{v}$, and $\tilde{\sigma}$ are calculated.

In addition to comparing the observables $m^{x}(t)$ and $S_{12}^{x x}(t)$, we provide a quantitative measure of the accuracy of each method by calculating the trace distance between the EXACT reduced density matrix at each time step and the corresponding reduced density matrix calculated using the different TN methods. In particular, we find the trace distance $T_{2}(t)$ of the nearest-neighbor reduced density matrices $T_{2}\left(\rho_{j l}, \phi_{j l}\right)=\frac{1}{2} \operatorname{tr}\left(\sqrt{\left(\rho_{j l}-\phi_{j l}\right)^{\dagger}\left(\rho_{j l}-\phi_{j l}\right)}\right)$, where $T_{2}(t)$ is averaged over the four nearest-neighbor reduced density matrices of the two-site unit cell. By observing $m^{x}(t)$, and $S_{12}^{x x}$ and the trace distance $T_{2}$ in Figs. 5(a)-5(c), it is clear that the SU method does not reproduce the EXACT results to the same accuracy as FET + WTG. Figure 5(a) and its inset demonstrate that, while FET + WTG shows clear systematic improvement in accuracy as $D$ is increased, SU shows only minor and not clearly systematic reduction in $T_{2}(t \gamma=10)$ even if $D$ is increased well beyond that for which FET + WTG demonstrates good convergence. For values of $D>3, T_{2}$ is consistently about an order of magnitude smaller for FET + WTG than for SU, demonstrating the improved compression and greater accuracy of WTG + FET. The observables in Figs. 5(b) and 5(c) calculated using SU deviate from the
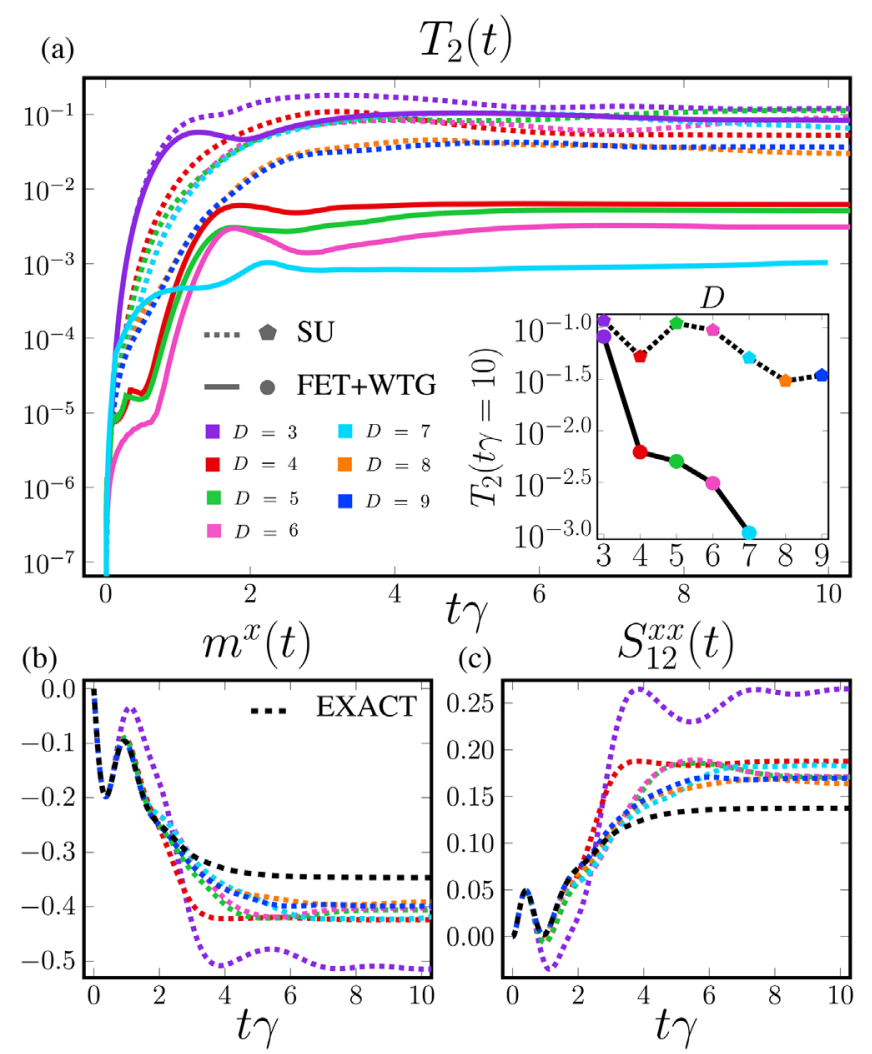

FIG. 5. Comparison between FET + WTG (solid lines), SU (dotted lines), and EXACT (dashed lines) in the moderately damped regime of the dissipative Ising model $V / \gamma=1.2$, $h^{x} / \gamma=0.0$. (a) Trace distance $T_{2}(t)$ as a function of time and at $t \gamma=10$ (inset) for a range of bond dimensions. (b) Magnetization $m^{x}(t)$ and (c) nearest-neighbor $S_{12}^{x x}$, showing that FET + WTG outperforms SU.

EXACT dynamics considerably compared to FET + WTG [compare to Fig. 4(b)]; at times $t \gamma \gtrsim 2$, the SU method struggles to accurately capture the EXACT dynamics for all bond dimensions shown.

Finally, we compare how the two algorithms deal with internal correlations in the network and compare the fidelity of truncation at each time step. TNs with closed loops (or cyclic TNs) can suffer from an accumulation of internal correlations, which do not contribute to any property of the quantum state. To achieve an optimal TN representation of the state at each truncation step, it is necessary to remove these internal correlations. Furthermore, a buildup of these correlations can lead to problems in computation and breakdown of algorithms [72]. The cycle entropy $S_{\text {cycle }}$ defined in Ref. [72] prescribes a way of quantifying the extent of internal correlations in the network and is conveniently expressed in terms of the bond environment; details of its calculation in the present case are given in Appendix E. The cycle entropy $S_{\text {cycle }}$ plotted in Fig. 6(a) shows the extent of internal correlations in the network as a function of time. Initially, the network, which represents a product state, has no 

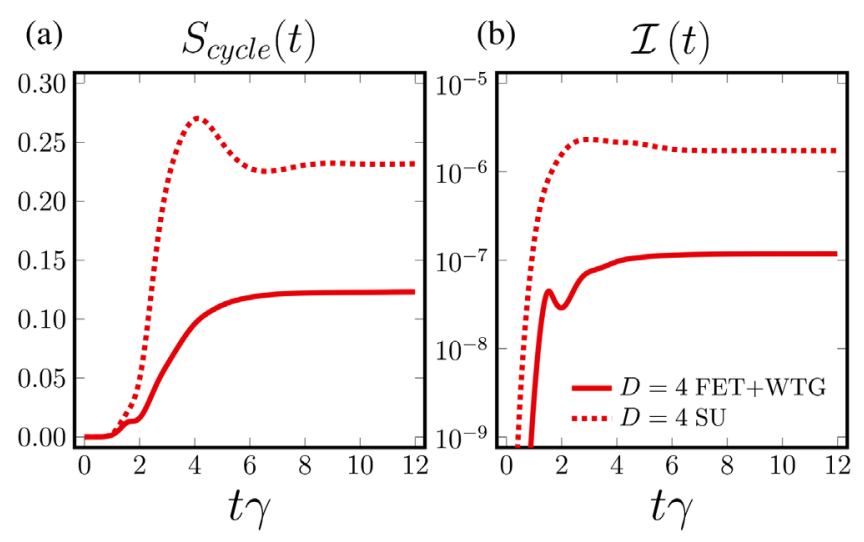

FIG. 6. (a) Accumulation of internal correlations in time quantified by the cycle entropy $S_{\text {cycle }}(t)$, which is more effectively curtailed by FET + WTG. (b) Infidelity of truncation $\mathcal{I}(t)$, which is an order of magnitude smaller than SU at each truncation step. The results form a moderately damped regime of the dissipative Ising model $V / \gamma=1.2, h^{x} / \gamma=0.0$ with $D=4$.

internal correlations. In time, the extent of internal correlations grows and saturates at a finite value. Importantly, $S_{\text {cycle }}$ grows more slowly and saturates at a smaller value for FET + WTG than it does for SU, illustrating that the proper truncation of bonds reduces the extent of internal correlations in the network. Although the growth of $S_{\text {cycle }}$ in this case is relatively benign, the failure of SU to curtail the accumulation of internal correlations may contribute to the breakdown of the algorithm in some circumstances. As a final comparison, we plot the infidelity of truncation $\mathcal{I}$ as a function of time for the two different methods in Fig. 6(b) and find that the FET + WTG method outperforms $\mathrm{SU}$, decreasing the infidelity between truncated and untruncated bonds by approximately an order of magnitude. Although the variational degree of the ansatz is the same in each case - they have the same $D$ and $\chi$ - the method by which enlarged bonds are truncated is crucially important in finding an optimal representation, thereby reducing the accumulation of errors due to inadequate truncation and ultimately giving the most accurate results.

\section{Driven-dissipative hard-code boson model}

In driven-dissipative quantum lattice models, dissipation to the bath is replenished via a coherent or incoherent drive. Driven-dissipative systems constitute an important class of models with direct relevance to experimental platforms such as driven coupled photon arrays in a variety of architectures [84]. In this section, we calculate steady-state properties of a driven-dissipative hard-core boson model that can be mapped to a lattice of interacting spin- $1 / 2$ particles. The Hamiltonian is given in the rotating frame by

$$
\hat{H}=\sum_{j}\left[-\Delta \hat{\sigma}_{j}^{+} \hat{\sigma}_{j}^{-}+F\left(\hat{\sigma}_{j}^{+}+\hat{\sigma}_{j}^{-}\right)\right]-\frac{\mathrm{J}}{z} \sum_{\langle j, l\rangle} \hat{\sigma}_{j}^{+} \hat{\sigma}_{l}^{-},
$$

where $\Delta=\omega_{p}-\omega_{c}$ is the detuning between the pump frequency $\omega_{p}$ and the on-site energy $\omega_{c}, F$ is the pump field strength, $J$ is the hopping coupling, and the sum $\sum_{\langle j, l\rangle}$ runs over nearest neighbors in the lattice of coordination number $z$. The spins undergo dissipation at a rate $\gamma$ described by a Lindblad operator $\hat{L}_{j}=\sqrt{\gamma} \hat{\sigma}_{j}^{-}$, which is the same at each site, where the spin-raising and spin-lowering operators are defined as $\hat{\sigma}^{ \pm} \equiv \frac{1}{2}\left(\hat{\sigma}^{x} \pm i \hat{\sigma}^{y}\right)$.

We compare steady-state expectation values with those calculated using the corner-space renormalization method [56]. To this end, we consider an array of hard-core bosons with $\Delta / \gamma=5, F / \gamma=2$, and $J / \gamma=1$ and calculate the average single-site boson density $n=1 / 2\left(n_{j}+n_{l}\right)$, the nearest-neighbor $(\langle j, l\rangle)$ correlation functions $g^{(2)}$ averaged over all combinations of $(\langle j, l\rangle)$, where

$$
g_{j, l}^{(2)}=\frac{\left\langle\hat{\sigma}_{j}^{+} \hat{\sigma}_{l}^{+} \hat{\sigma}_{j}^{-} \hat{\sigma}_{l}^{-}\right\rangle}{\left\langle\hat{\sigma}_{j}^{+} \hat{\sigma}_{j}^{-}\right\rangle\left\langle\hat{\sigma}_{l}^{+} \hat{\sigma}_{l}^{-}\right\rangle}, \quad n_{j}=\operatorname{tr}\left(\hat{\sigma}_{j}^{+} \hat{\sigma}_{j}^{-} \rho_{s s}\right),
$$

and the average real part of $\operatorname{Re}\left[\operatorname{tr}\left(\hat{\sigma}^{-} \rho_{s s}\right)\right]$ at each lattice site.

Staring from an initial product state, we find the steady state for a set of parameters $D, \chi$, and $\epsilon_{D^{\prime}}$, where convergence in time is achieved when all expectation values $\hat{o}$ up to the next-nearest neighbor fulfill a convergence criterion of $\epsilon_{t}<10^{-6}$, where

$$
\epsilon_{t}=\frac{\left|\operatorname{tr}\left(\hat{o} \rho_{t+\tau}\right)-\operatorname{tr}\left(\hat{o} \rho_{t}\right)\right|}{\left|\operatorname{tr}\left(\hat{o} \rho_{t}\right)\right| \tau} .
$$

We use the steady-state iPEPO calculated for one set of variational parameters as an initial state for the next until convergence to the desired precision is achieved. Results of this procedure are given in Table I, along with comparable results from Ref. [56].

The steady-state values converge as the iPEPO variational parameters are increased, and they are comparable to the results of the corner-space renormalization method. Where we might expect increasing the corner-space renormalization method lattice size $N_{x} \times N_{y}$ will give results closer to the FET + WTG method, which represents the thermodynamic limit directly, we find that the opposite is true, with a lattice size of $4 \times 4$ closer in agreement to FET + WTG than $8 \times 8$. This discrepancy could be due to finite-size effects or translational symmetry breaking, which may be present in the $N_{x} \times N_{y}$ results, and is not observed in the iPEPO solution where we have enforced two-site translational invariance by choosing a two-site unit cell. Translational symmetry breaking could arise due to a tunneling-induced instability, as was observed in Refs. [24,40]; this could be investigated using the iPEPO method by comparing the results of Table I to equivalent ones calculated using an iPEPO ansatz with a larger unit cell. Alternatively, the discrepancy might arise from a lack of convergence in the finite system size of the corner-space 
TABLE I. Steady-state values of a hard-core boson model on an infinite square lattice with parameters $\Delta / \gamma=5.0, F / \gamma=2.0$, and $J / \gamma=1.0$ calculated using FET + WTG. In each case, we use a time step of $\tau \gamma=0.0025$. For comparison, we tabulate results for the same parameters from the corner-space renormalization method [56] for different sizes $N_{x} \times N_{y}$.

\begin{tabular}{|c|c|c|c|c|c|}
\hline \multicolumn{6}{|c|}{$J / \gamma=1.0 F / \gamma=2.0 \Delta / \gamma=5.0$} \\
\hline$D$ & $\chi$ & $\epsilon_{D^{\prime}}$ & $n$ & $\operatorname{Re}\left(\left\langle\hat{\sigma}^{-}\right\rangle\right)$ & $g_{\langle j, l\rangle}^{(2)}$ \\
\hline$\overline{1}$ & 1 & $10^{-6}$ & 0.09482 & 0.27619 & 1.0 \\
\hline \multirow[t]{5}{*}{3} & 9 & $10^{-4}$ & 0.09545 & 0.27674 & 1.06243 \\
\hline & 9 & $10^{-5}$ & 0.09534 & 0.27680 & 1.06353 \\
\hline & 9 & $10^{-6}$ & 0.09534 & 0.27681 & 1.06360 \\
\hline & 9 & $10^{-7}$ & 0.09535 & 0.27680 & 1.06344 \\
\hline & 15 & $10^{-7}$ & 0.09535 & 0.27680 & 1.06344 \\
\hline \multirow[t]{2}{*}{4} & 8 & $10^{-7}$ & 0.09548 & 0.27670 & 1.06440 \\
\hline & 12 & $10^{-7}$ & 0.09548 & 0.27670 & 1.06443 \\
\hline \multirow[t]{2}{*}{5} & 10 & $10^{-7}$ & 0.09548 & 0.27670 & 1.06443 \\
\hline & 15 & $10^{-7}$ & 0.09548 & 0.27670 & 1.06443 \\
\hline \multicolumn{6}{|c|}{$N_{x} \times N_{y}$ Corner-space renormalization method } \\
\hline $4 \times 4$ & & & $0.0954(1)$ & $0.2764(2)$ & $1.0643(3)$ \\
\hline $8 \times 4$ & & & $0.09527(2)$ & $\ldots$ & $1.0436(3)$ \\
\hline $8 \times 8$ & & & $0.0948(2)$ & $\ldots$ & $1.0237(6)$ \\
\hline
\end{tabular}

renormalization method. Results concerning a drivendissipative Bose-Hubbard model on a two-dimensional Lieb lattice are presented in Table II of Ref. [48] and are calculated using a positive-P algorithm: a method that can be considered to produce the exact solution when the associated numerics remain stable. Those results suggest that quite large lattices-greater than or approximately $10 \times 10$ unit cells - are required to achieve convergence. Nevertheless, the precise origin of this discrepancy is left for future work.

\section{Anisotropic dissipative $X Y$ model}

Having demonstrated the capabilities of the algorithm, by choosing an interesting example, we now show that the method is highly suitable to address physical questions. In particular, two-dimensional systems can host a unique set of phenomena; here, we explore the stability with respect to fluctuations of a spontaneously symmetry-broken staggered- $X Y$ (s $X Y$ ) phase in the steady state of an anisotropic dissipative $X Y$ model. While the mean-field theory predicts that the $\mathrm{s} X Y$ phase is stable in two dimensions, it is not clear whether it remains accessible if fluctuations at the microscopic level are accounted for and if any long-range order associated with the $\mathrm{s} X Y$ phase is present. The anisotropic dissipative $X Y$ model has a Hamiltonian of the form

$$
\hat{H}=\frac{J}{z} \sum_{\langle j, k\rangle} \hat{\sigma}_{j}^{x} \hat{\sigma}_{k}^{x}-\hat{\sigma}_{j}^{y} \hat{\sigma}_{k}^{y}
$$
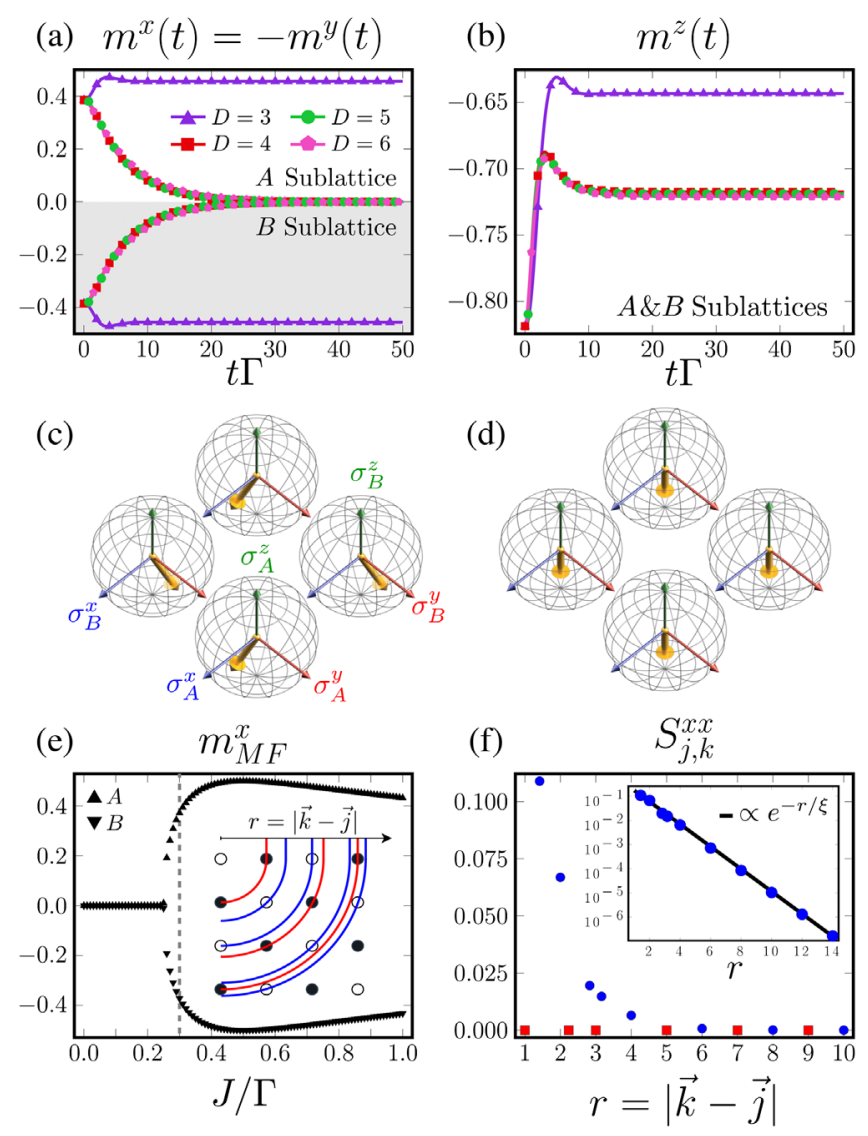

FIG. 7. Fate of staggered- $X Y$ phase at $J / \Gamma=0.3$. (a,b) Local magnetizations $m^{x, y, z}(t)$ on the $A$ and $B$ sublattices as the state evolves from the $D=1$ steady-state solution for bond dimensions $D \in[3,4,5,6]$. (c,d) Representation of a $2 \times 2$ plaquette of the lattice in (c) the staggered- $X Y$ phase $(D=1$ steady state) and (d) the uniform phase ( $D=6$ steady state). (e) Mean-field phase diagram with a transition at $J / \Gamma=\frac{1}{4}$. Inset: radii $r=|\vec{k}-\vec{j}|$ of an odd (red) and even (blue) number of steps on the lattice. (f) Correlation function $S_{j, k}^{x x}=\left\langle\sigma_{j}^{x} \sigma_{k}^{x}\right\rangle$ versus distance $r$, which has a staggered form that is a remnant of the staggered- $X Y$ phase. Correlations at odd step radii (red squares) are zero, and those at even step radii (blue circles) are finite and decay with $r$. Inset: exponential fit to even step correlations, giving a correlation length $\xi \approx 0.93$.

with a nearest-neighbor hopping $J$ and coordination number $z=4$, as well as dissipation described by local Lindblad operators $\hat{L}_{j}=\sqrt{\Gamma} \hat{\sigma}_{j}^{-}$at each lattice site. The (Gutzwiller) mean-field phase diagram, plotted in Fig. 7(e), was studied in Ref. [85] and shows that, for $J / \Gamma>1 / 4$, the steady state hosts a staggered- $X Y$ symmetry-broken phase in which the spins divide into $A$ and $B$ sublattices with angles $\pm \theta$ relative to the $x=y$ line on the Bloch sphere, as depicted in Fig. 7(c). The spontaneous breaking of this continuous $U(1)$ symmetry means that $\theta$ can take any value and allows for vortexlike topological defects in the lattice. The question of whether or not the $\mathrm{s} X Y$ phase is accessible in two dimensions if corrections beyond mean-field theory are accounted 
for has previously been addressed using a Keldysh field theory approach $[85,86]$. In this approach, an effective model is constructed by mapping the spins to bosons, an approach that does not capture the microscopic physics of the spin model but addresses the behavior in the longwavelength limit. In that approximation, it was found that the steady-state physics of the effective model is described by a partition function in the same universality class as the classical $X Y$ model, and therefore, one should expect a Kosterlitz Thouless transition in two dimensions. However, it was also predicted in Ref. [86], based on a simple meanfield theory analysis, that the effective temperature of the model will be greater than the Kosterlitz Thouless temperature, such that the ordered phase will not be accessible when quantum fluctuations are included and any long-range algebraic order will be absent or at least significantly diminished. We can now use our method to address this question exactly by directly solving the microscopic spin model close to the transition point $J / \Gamma=1 / 4$, where the mean-field theory is expected to break down. Moreover, we are able to give a quantitative picture of the system by calculating not only local observables as a function of time but also spatial correlation functions in the steady state.

We first find the steady-state iPEPO representation of the model for a bond dimension $D=1$ —equivalent to a meanfield solution-at $J / \Gamma=0.3$, which lies just within the $\mathrm{s} X Y$ phase. To do this, we initialize the iPEPO in a state for which the symmetry is explicitly broken, $\left\langle\sigma_{A}^{x}\right\rangle=-\left\langle\sigma_{B}^{x}\right\rangle=1$, and calculate the $D=1$ steady state with WTG + FET. Then, using the symmetry-broken $D=1 \mathrm{iPEPO}$ solution as an initial state, we systematically add quantum fluctuations by calculating steady states for bond dimensions $D \in$ $[3,4,5,6]$ until convergence. Results of this procedure are presented in Fig. 7. For bond dimensions $D=3$, we find that the system remains in the s $X Y$ phase. For $D \in[4,5,6]$, however, the spin magnetization $m^{z}(t)$, which is uniform across the lattice, is slightly modified, and the magnetizations $m^{x}(t)$ and $m^{y}(t)$ on each sublattice slowly tend towards zero such that the continuous symmetry no longer appears broken-depicted in Fig. 7(d). Therefore, the mean-field $\mathrm{s} X Y$ phase is unstable to fluctuations, corroborating the Keldysh field theory predictions of Ref. [86]. This proves that long-wavelength fluctuations captured by the approximate theory dominate over other microscopic fluctuations. In Fig. 7(f), we plot the correlation function $S_{k, j}^{x x}=\left\langle\sigma_{j}^{x} \sigma_{k}^{x}\right\rangle$ (note that $\left\langle\sigma_{j}^{x}\right\rangle\left\langle\sigma_{k}^{x}\right\rangle \approx 0$ ), which shows a staggered structure reminiscent of the $\mathrm{s} X Y$ phase where correlations at a radii $r$ [see Fig. 7(e) inset] corresponding to an odd number of steps on the lattice are zero, whereas even step correlations are finite and decay with $r$. Considering only the even step correlations in the inset of Fig. 7(f), we find that the decay is well approximated by an exponential function of the form $S_{r \in \text { even }}^{x x} \propto e^{-r / \xi}$, with $\xi \approx 0.93$; any long-range algebraic order that may have been associated with the symmetry-broken phase is not present in the iPEPO solution, suggesting that the system is in the disordered phase. Good convergence is found for $D=6$ and $\tau \Gamma=0.01$, resulting in infidelity of truncation $\mathcal{I}(t)<10^{-9}$.

\section{DISCUSSION}

We have developed a new TN algorithm capable of accurately simulating dynamics of dissipative quantum lattice models on a two-dimensional square lattice directly in the thermodynamic limit. The method adapts the FET and WTG fixing techniques of Ref. [72] to the iPEPO TN ansatz for mixed states. Comparisons with exact numerical results demonstrate an excellent accuracy of the method and its performance across different dissipative regimes. Contrasting with the more efficient, but much less accurate, simple update truncation scheme, we have proven that it is necessary to optimally truncate enlarged bonds to obtain accurate results. We have shown the applicability of the technique for calculating steady-state properties of drivendissipative systems by comparison with results in the literature. The method performs well in regimes where mean-field approximation fails, proving able to capture substantial correlations in the presence of dissipation. Finally, we have shown that a staggered- $X Y$ phase of the dissipative anisotropic $X Y$ model predicted by mean-field theory is not stable if correlations are included; while a remnant of the staggered structure remains in the correlation function, its decay is well approximated by an exponential function, and no long-range order remains.

As with similar algorithms for iPEPS, the principal contribution to the computational complexity of the algorithm comes from the calculation of the effective environment, which is updated at each time step (here using CTMRG). The leading cost of the version of CTMRG we use arises from a singular value decomposition of order $O\left(\chi_{\mathrm{HS}}^{3} D^{6}\right)$; improvements in performance can therefore be achieved by optimizing this step-for instance, using a fixed-point method such as the FPCM [80] or approximating the effective environment by using a boundary matrix product state to represent the boundary of the system. Numerous algorithms have been developed to calculate the fixed point, including a TEBD $[87,88]$ or variational MPStangent space methods (VUMPS) [80,89-92], and they can lead to significant speedup for TNs that are close to being critical [80].

In addition to accurately determining steady-state properties such as long-range, equal-time, correlation functions, this work facilitates the calculation of more complex dynamical properties, e.g., dynamical correlation functions and fluorescence spectra of strongly correlated, drivendissipative quantum lattice models. A significant advantage of both the FET method of truncating enlarged bonds and the WTG method of fixing the TN gauge is that they can be used in tensor networks of arbitrary geometries, provided the bond environment can be calculated efficiently. In this 
regard, straightforward adaptations of the method we have presented in this work could be used to treat drivendissipative models with longer-range interactions or those defined on more complicated network structures such as hyperbolic lattices [5], as well as problems related to functional quantum biology $[93,94]$.

\section{ACKNOWLEDGMENTS}

M. H.S. gratefully acknowledges financial support from EPSRC (Grants No. EP/R04399X/1 and No. EP/ K003623/2). This work was supported by the Engineering and Physical Sciences Research Council (Grant No. EP/ L015242/1).

\section{APPENDIX A: CALCULATING THE EFFECTIVE ENVIRONMENTS}

Given tensors representing the unit cell of the 2D lattice, we calculate the effective environments $\mathcal{E}^{\text {tr }}$ and $\mathcal{E}^{\mathrm{HS}}$ using a variant of the corner transfer matrix renormalization group (CTMRG) algorithm [74-80]. To improve stability and convergence properties of the CTMRG algorithm, as well as the conditioning of the bond environment $\Upsilon_{j l}$, we find it helpful to use the variant of CTMRG presented in Ref. [80], which makes use of an intermediate singular value decomposition. Following Ref. [80], we refer to Fig. 8 in describing the basic steps involved in the leftmove component of the CTMRG algorithm used for calculating $\mathcal{E}^{\mathrm{HS}}$ for an iPEPO with a two-site unit cell. This algorithm goes as follow: Consider the unvectorized sixth-rank iPEPO tensors $A_{j}$ and $A_{l}$.

(i) To calculate the trace effective environment $\mathcal{E}^{\text {tr }}$, trace over the physical dimensions of the iPEPO unit-cell tensors, giving the fourth-rank tensors $a \rightarrow \operatorname{tr}_{d}\left(A_{j}\right)$ and $b \rightarrow \operatorname{tr}_{d}\left(A_{l}\right)$, where we have split the bond matrix in two [Figs. 3(a) and 3(b)] and contracted each half with $a$ and $b$ appropriately.

(ii) Alternatively, to calculate the Hilbert-Schmidt effective environment $\mathcal{E}^{\mathrm{hs}}$, first find the Hilbert-Schmidt inner product over the physical indices of the vectorized $A_{j}$ and $A_{l}$, giving the eighth-rank tensors $a \rightarrow \operatorname{tr}_{d}\left(A_{j} A_{j}^{\dagger}\right)$ and $b \rightarrow \operatorname{tr}_{d}\left(A_{l} A_{l}^{\dagger}\right)$ [Fig. 3(c)].

(iii) The left-move CTMRG step then proceeds as follows, where the tensor diagrams of Fig. 8 show the eighth-rank versions of $a$ and $b$ and therefore represent steps in the calculation of $\mathcal{E}^{\mathrm{HS}}$.

(iv) (a) Construct the upper- and lower-half system transfer matrices and take a SVD to find the upper and lower decompositions $U u_{a} S u_{a b} V u_{b}^{\dagger}$ and $U d_{a} S d_{a b} V d_{b}^{\dagger}$.

(v) (b) Define $F l u_{a} \equiv U u_{a} S u_{a b}^{1 / 2}, F r u_{a} \equiv S u_{a b}^{1 / 2} V u_{a}^{\dagger}$, $F l d_{a} \equiv U d_{a} S d_{a b}^{1 / 2}$, and $F r d_{a} \equiv S d_{a b}^{1 / 2} V d_{a}^{\dagger}$, where singular values of magnitudes (relative to the largest (a)

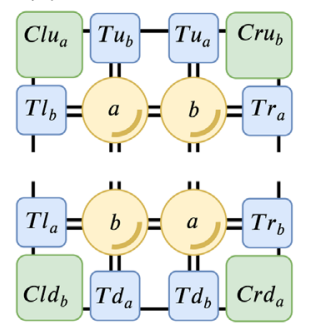

(b)

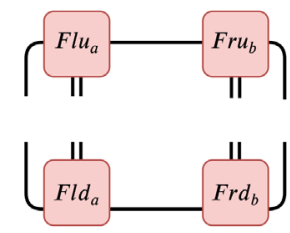

(d)

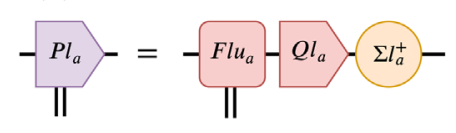

(f)
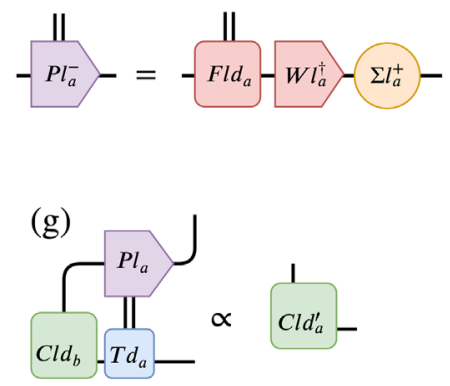

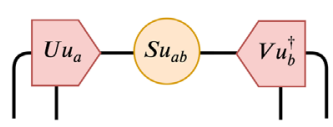

$\approx$

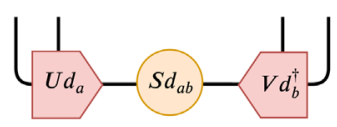

(c)

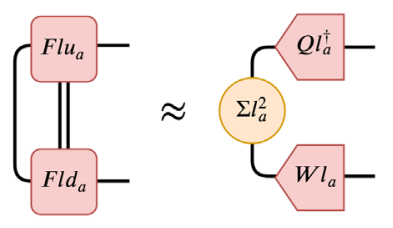

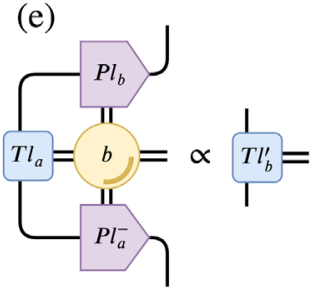

(h)

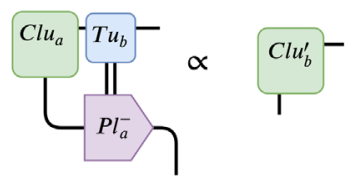

FIG. 8. Tensor diagrams representing some of the steps involved in performing the left-move component of the CTMRG algorithm used to calculate the effective environment $\mathcal{E}^{\mathrm{HS}}$.

singular value) less than some small tolerance are truncated to improve stability.

(vi) (c) Use the so-called biorthogonalization procedure (see Ref. [80] for further details) to calculate $P l$ and $\mathrm{Pl}^{-}$, the first step of which is to contract $\mathrm{Flu}_{a}$ with $F l d_{a}$ and perform a SVD to find $W l_{a}, Q l_{a}$, and the diagonal matrix $\Sigma l_{a}^{2}$.

(vii) (d,f) Calculate the projectors $P l_{a}=F l u_{a} Q l_{a} \Sigma l_{a}^{+}$ and $P l_{a}^{-}=F l d_{a} W l_{a}^{\dagger} \Sigma l_{a}^{+}$, with $\Sigma l^{+}$being the MoorePenrose pseudoinverse of $\Sigma l$.

(viii) (e,h) Repeat steps (a)-(f) to calculate $P l_{b}$ and $P l_{b}^{-}$ by replacing $a \leftrightarrow b$ in the upper- and lower-half system transfer matrices. Using these projectors, the updated environment tensors $T l_{b}^{\prime}, T l_{a}^{\prime}, C l u_{a}^{\prime}$, $C l u_{b}^{\prime}, C l d_{a}^{\prime}$, and $C l d_{b}^{\prime}$ are calculated and normalized as shown in Figs. 8(h), 8(j), and 8(k). This is one iteration of the left-move component of this CTMRG algorithm. 
Steps (iv) through to (viii) describe one interaction of the left-move subroutine of the CTMRG algorithm.

A similar sequence of steps is used to perform the rightmove, up-move, and down-move steps in CTMRG. The set of directional moves is repeated in series until the vectors of singular values of the corner transfer matrices converge. It is possible to perform the right-move step at the same time as the left-move one by following the biorthogonalization routine starting with $\mathrm{Fru}_{b}$ and $\mathrm{Frd}_{b}$ calculated in step (b) above, and similarly for the up-move and down-move steps.

\section{APPENDIX B: FULL ENVIRONMENT TRUNCATION}

An adapted FET algorithm [72] is used to truncate enlarge bonds of the iPEPO as follows. Let the state of the full system at time $t$ be $\rho_{t}$ and calculate the Hilbert-Schmidt environment $\mathcal{E}_{j, l}^{\mathrm{HS}}$ of the iPEPO representing $\rho_{t}$ as discussed in Appendix A. Find $A_{j}^{\prime}$ and $A_{l}^{\prime}$ by applying the Trotterized dynamical map

(a)

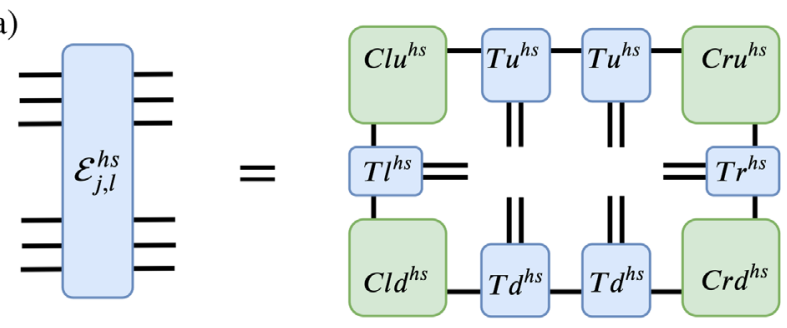

(b)

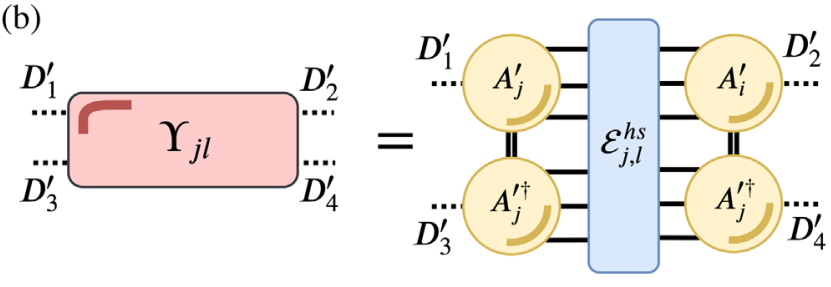

(c)

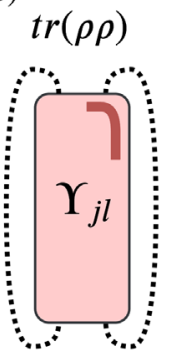

(d)

$\operatorname{tr}(\phi \phi)$

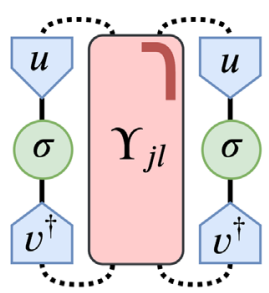

(e)

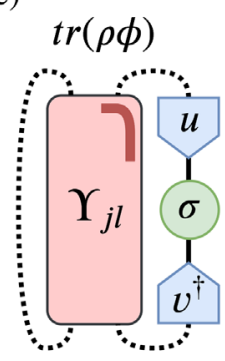

FIG. 9. (a) Effective environment $\mathcal{E}_{j, l}^{\mathrm{HS}}$ of the tensors at neighboring sites $j$ and $l$. (b) Bond environment $\Upsilon_{j, l}$, which is the contraction of $\mathcal{E}_{j, l}^{\mathrm{HS}}$ and the updated tensors $A_{j}^{\prime}$ and $A_{l}^{\prime}$ with enlarged bonds $\left\{D_{j}^{\prime}\right\} \geq D$. In panels (c)-(e), using $\Upsilon_{j, l}$, the terms in the fidelity between the truncated $(\phi)$ and untruncated $(\rho)$ density matrices are calculated by contracting with the isometries $u, v$ and the bond matrix $\sigma$. and decompose the result via SVD, retaining the $D^{\prime}$ singular values with a magnitude (relative to the largest singular value) greater than $\epsilon_{D^{\prime}}$. Contract $A_{j}^{\prime}$ and $A_{l}^{\prime}$ with the effective environment $\mathcal{E}_{j, l}^{\mathrm{HS}}$, leaving only the enlarged bonds uncontracted, as illustrated in Fig. 9(d). This procedure leaves us with the fourth-rank bond environment tensor $\Upsilon_{j l}$. Using the bond environment $\Upsilon_{i j}$, the tensors involved in the Rayleigh quotient proportional to $\mathcal{F}^{2}$ are calculated. Figures 9(e)-9(g) illustrate the tensor contractions required to construct $\operatorname{tr}(\rho \phi)$, $\operatorname{tr}(\phi \phi)$, and $\operatorname{tr}(\rho \rho)$, allowing us to represent $\mathcal{F}^{2}(\rho, \phi) \operatorname{tr}(\rho \rho)$ in terms of the isometries $u$ and $v$, the bond matrix $\sigma$, and the bond environment $\Upsilon_{i j}$, where we note that the term $\operatorname{tr}(\rho \rho)$ is independent of $u, \sigma$, and $v$.

The alternating optimization of $u, v$, and $\sigma$ proceeds as follows and is illustrated in Fig. 10. Defining $R \equiv \sigma v$ [Fig. 10(c)], the $R_{m}$ that maximizes $\mathcal{F}^{2}(\rho, \phi) \operatorname{tr}\left(\rho^{2}\right)$ [Fig. 10(a)] is found by keeping $v$ fixed and solving a generalized eigenvalue problem in $R$ (see Appendix $C$ for further details). The updated tensors $\sigma^{\prime}$ and $u^{\prime}$ are then calculated using a SVD as illustrated in Fig. 10(e). Similarly, by defining $L \equiv v^{\prime} \sigma^{\prime}$, the optimal $L_{m}$ is found, giving $u^{\prime \prime}, \sigma^{\prime \prime}$, and $v^{\prime \prime}$. The alternating process is repeated until the convergence of $\tilde{u}, \tilde{\sigma}$, and $\tilde{v}$ isometries is reached.

(a)

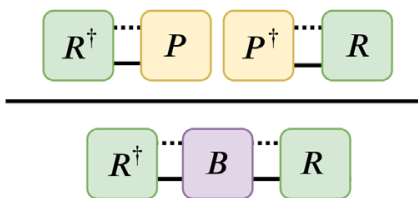

$\propto$

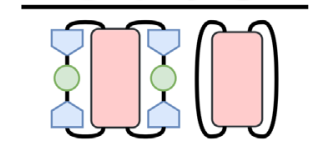

(b)

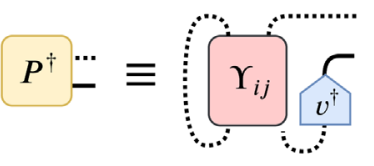

(c)

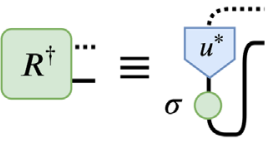

(d)

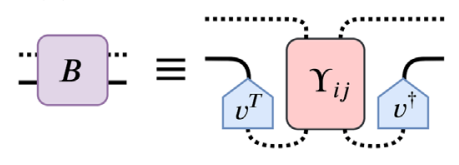

(e)

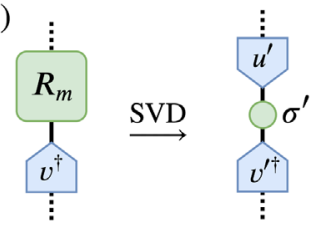

FIG. 10. Tensor diagrams representing some of the steps involved in finding the isometries $\tilde{u}$ and $\tilde{v}$ and the bond matrix $\tilde{\sigma}$, which maximize the fidelity between the truncated and untruncated bonds. In panel (a), the Rayleigh quotient in $R$ is proportional to $\mathcal{F}^{2}$. Panel (b) shows that $P$ is the contraction of the bond environment $\epsilon_{j, l}$ and the isometry $v$. In panel (c), we show that $R$ is the contraction of the bond matrix $\sigma$ and the isometry $u$. Panel (d) shows that $B$ is the contraction of $\Upsilon_{j l}$ with the isometry $v$. In panel (e), the new (primed) isometries are found by singular value decomposition of the contraction of the maximal eigenvector $R_{m}$ and $v$. 


\section{APPENDIX C: OPTIMIZING RAYLEIGH QUOTIENT}

A Rayleigh quotient of the form $F(R)=\left(\vec{R}^{\dagger} A \vec{R} / \vec{R}^{\dagger} B \vec{R}\right)$ is maximized by the eigenvector $\vec{R}_{m}$, which corresponds to the largest eigenvalue $\lambda_{m}$ of the generalized eigenvalue problem $A \vec{R}_{i}=\lambda_{i} B \vec{R}_{i}$. Since the matrix $A$ is constructed as an outer product $A=\vec{P}^{\dagger} \vec{P}$, the $\overrightarrow{R_{m}}$ that maximizes the Rayleigh quotient is given by $\overrightarrow{R_{m}}=\vec{P} B^{-1}$. In practice, it is possible to calculate $\overrightarrow{R_{m}}$ directly by inverting $B$ or by solving the system of linear equations $\overrightarrow{R_{m}} B=\vec{P}$ using, for example, a linear regression algorithm. Care must be taken at this stage to maintain the stability of the algorithm. If solving by direct inversion, we find it advantageous to either use a Moore-Penrose pseudoinverse [95] with some tolerance or solve via linear regression with an intermediate truncated singular value decomposition. In our simulations, we maximize the Rayleigh quotient by instead solving the generalized eigenvalue problem $A \vec{R}=\lambda B \vec{R}$ either by full diagonalization or by iterative methods to calculate only the maximal eigenvector $\overrightarrow{R_{m}}$ (Lanczos or Arnoldi).

\section{APPENDIX D: EXACT SOLUTION OF DISSIPATIVE ISING MODEL}

In order to provide a benchmark for our new TN method, we solve the dissipative transverse Ising model in Sec. III A using the method of Ref. [83], which we briefly describe. As shown in Ref. [83], if a Liouvillian is structured such that coherences are not mapped to populations (and vice versa), then correlations in a system remain localized. This structure allows for an efficient exact determination of the time evolution of the local observables, which initially only have support on a suitably small sublattice. In particular, an observable $O(t)$, which initially has support on a set of lattice sites $\mathcal{A}$, can be calculated at all times by solving, in the Schrodinger picture,

$$
O(t)=\operatorname{tr}_{\mathcal{A} \cup \mathcal{B}}\left[\hat{O} \exp \left(t \mathcal{L}_{\mathcal{A B}}\right) \hat{\rho}_{\mathcal{A B}}\right]
$$

where $\mathcal{B}$ is the set of lattice sites that are nearest neighbors of $\mathcal{A}$ and for which the Hamiltonian has simultaneous support on $\mathcal{A}$ and $\mathcal{B}$.

We choose to calculate up to next-nearest-neighbor (in a lattice row or column) correlations $S_{j l}^{x x}(t)$ in time and therefore choose as $\mathcal{A}$ the set of three contiguous lattice sites in a row (in either the $x$ or $y$ lattice dimension) of the infinite two-dimensional lattice. For a two-local Liouvillian, $\mathcal{B}$ is identified as the eight nearest-neighbor lattice sites of $\mathcal{A}$. Observables $O(t)$ can then be calculated efficiently by solving Eq. (D1) using standard techniques from quantum optics (we used the Julia package QuantumOptics.j1 [96] to calculate the exact results).

\section{APPENDIX E: CYCLE ENTROPY}

For closed systems, $S_{\text {cycle }}$ is defined as the von Neumann entropy of the normalized spectrum of a bond environment left contracted with the bond matrix $(\sigma \otimes \sigma) \Upsilon$, and it is constructed as an inner product of pure states (see Ref. [72] for details). Here, we instead use the bond environment left contracted with the bond matrix $(\sigma \otimes \sigma) \Upsilon$, which is constructed using $\mathcal{E}^{\mathrm{HS}}$ and which is defined in terms of mixed rather than pure states to calculate $S_{\text {cycle }}$,

$$
S_{\text {cycle }}=-\sum_{\alpha}\left(\tilde{\lambda}_{\alpha} \log _{2}\left(\tilde{\lambda}_{\alpha}\right)\right)
$$

where $\tilde{\lambda}_{\alpha} \equiv\left|\lambda_{\alpha}\right| /\left(\sum_{\alpha}\left|\lambda_{\alpha}\right|\right)$ are the absolute values of the eigenvalues of $(\sigma \otimes \sigma) \Upsilon$. A cycle entropy $S_{\text {cycle }} \approx 0$ indicates that there are no (or negligible) internal correlations associated with the bond environment, and in this case, an optimal or near-optimal truncation can be achieved by transforming to WTG and discarding small WTG coefficients. However, if $S_{\text {cycle }}$ is larger $\left(S_{\text {cycle }} \gtrsim 10^{-3}\right)$ (see Ref. [72]), such a straightforward truncation scheme will not give an optimal truncation, and internal correlations may accumulate as the algorithm progresses. We find that in most cases, when starting with a product state, $S_{\text {cycle }}$ quickly increases, and the FET scheme is required.

[1] H. Walther, B. T. H. Varcoe, B.-G. Englert, and T. Becker, Cavity Quantum Electrodynamics, Rep. Prog. Phys. 69, 1325 (2006).

[2] A. Reiserer and G. Rempe, Cavity-Based Quantum Networks with Single Atoms and Optical Photons, Rev. Mod. Phys. 87, 1379 (2015).

[3] S. Schmidt and J. Koch, Circuit QED Lattices: Towards Quantum Simulation with Superconducting Circuits, Ann. Phys. (Amsterdam) 525, 395 (2013).

[4] A. A. Houck, H. E. Türeci, and J. Koch, On-Chip Quantum Simulation with Superconducting Circuits, Nat. Phys. 8, 292 (2012).

[5] A. J. Kollár, M. Fitzpatrick, and A. A. Houck, Hyperbolic Lattices in Circuit Quantum Electrodynamics, Nature (London) 571, 45 (2019).

[6] I. Carusotto, A. A. Houck, A. J. Kollár, P. Roushan, D. I. Schuster, and J. Simon, Photonic Materials in Circuit Quantum Electrodynamics, Nat. Phys. 16, 268 (2020).

[7] I. Carusotto, D. Gerace, H. E. Tureci, S. De Liberato, C. Ciuti, and A. Imamoğlu, Fermionized Photons in an Array of Driven Dissipative Nonlinear Cavities, Phys. Rev. Lett. 103, 033601 (2009).

[8] R. O. Umucalilar and I. Carusotto, Fractional Quantum Hall States of Photons in an Array of Dissipative Coupled Cavities, Phys. Rev. Lett. 108, 206809 (2012).

[9] T. Grujic, S. R. Clark, D. Jaksch, and D. G. Angelakis, Non-equilibrium Many-Body Effects in Driven Nonlinear Resonator Arrays, New J. Phys. 14, 103025 (2012). 
[10] J. Kasprzak, S. Reitzenstein, E. A. Muljarov, C. Kistner, C. Schneider, M. Strauss, S. Höfling, A. Forchel, and W. Langbein, Up on the Jaynes-Cummings Ladder of a Quantum-Dot/Microcavity System, Nat. Mater. 9, 304 (2010).

[11] L. Jin, M. Pfender, N. Aslam, P. Neumann, S. Yang, J. Wrachtrup, and R.-B. Liu, Proposal for a Room-Temperature Diamond Maser, Nat. Commun. 6, 8251 (2015).

[12] A. Amo and J. Bloch, Exciton-Polaritons in Lattices: A Non-linear Photonic Simulator, C.R. Phys. 17, 934 (2016).

[13] C. Schneider, K. Winkler, M. D. Fraser, M. Kamp, Y. Yamamoto, E. A. Ostrovskaya, and S. Hfling, ExcitonPolariton Trapping and Potential Landscape Engineering, Rep. Prog. Phys. 80, 016503 (2017).

[14] N. Y. Kim, K. Kusudo, C. Wu, N. Masumoto, A. Löffler, S. Höfling, N. Kumada, L. Worschech, A. Forchel, and Y. Yamamoto, Dynamical d-Wave Condensation of ExcitonPolaritons in a Two-Dimensional Square-Lattice Potential, Nat. Phys. 7, 681 (2011).

[15] D. Tanese, H. Flayac, D. Solnyshkov, A. Amo, A. Lemaître, E. Galopin, R. Braive, P. Senellart, I. Sagnes, G. Malpuech, and J. Bloch, Polariton Condensation in Solitonic Gap States in a One-Dimensional Periodic Potential, Nat. Commun. 4, 1749 (2013).

[16] F. Baboux, L. Ge, T. Jacqmin, M. Biondi, E. Galopin, A. Lemaître, L. Le Gratiet, I. Sagnes, S. Schmidt, H. E. Türeci, A. Amo, and J. Bloch, Bosonic Condensation and DisorderInduced Localization in a Flat Band, Phys. Rev. Lett. 116, 066402 (2016).

[17] S. Klembt, T. H. Harder, O. A. Egorov, K. Winkler, H. Suchomel, J. Beierlein, M. Emmerling, C. Schneider, and S. Höfling, Polariton Condensation in $S$ - and P-flatbands in a Two-Dimensional Lieb Lattice, Appl. Phys. Lett. 111, 231102 (2017).

[18] C. E. Whittaker, E. Cancellieri, P. M. Walker, D. R. Gulevich, H. Schomerus, D. Vaitiekus, B. Royall, D. M. Whittaker, E. Clarke, I. V. Iorsh, I. A. Shelykh, M. S. Skolnick, and D. N. Krizhanovskii, Exciton Polaritons in a Two-Dimensional Lieb Lattice with Spin-Orbit Coupling, Phys. Rev. Lett. 120, 097401 (2018).

[19] M. Dusel, S. Betzold, O. A. Egorov, S. Klembt, J. Ohmer, U. Fischer, S. Höfling, and C. Schneider, Room Temperature Organic Exciton-Polariton Condensate in a Lattice, Nat. Commun. 11, 2863 (2020).

[20] F. Brennecke, T. Donner, S. Ritter, T. Bourdel, M. Köhl, and T. Esslinger, Cavity QED with a Bose-Einstein Condensate, Nature (London) 450, 268 (2007).

[21] H.-P. Breuer et al., The Theory of Open Quantum Systems (Oxford University Press , 2002), https://doi.org/10.1093/ acprof:oso/9780199213900.001.0001.

[22] L. M. Sieberer, S. D. Huber, E. Altman, and S. Diehl, Dynamical Critical Phenomena in Driven-Dissipative Systems, Phys. Rev. Lett. 110, 195301 (2013).

[23] T. E. Lee, H. Häffner, and M. C. Cross, Collective Quantum Jumps of Rydberg Atoms, Phys. Rev. Lett. 108, 023602 (2012).

[24] J. Jin, A. Biella, O. Viyuela, L. Mazza, J. Keeling, R. Fazio, and D. Rossini, Cluster Mean-Field Approach to the Steady-State Phase Diagram of Dissipative Spin Systems, Phys. Rev. X 6, 031011 (2016).
[25] F. Nissen, S. Schmidt, M. Biondi, G. Blatter, H. E. Türeci, and J. Keeling, Nonequilibrium Dynamics of Coupled Qubit-Cavity Arrays, Phys. Rev. Lett. 108, 233603 (2012).

[26] J. Marino and S. Diehl, Driven Markovian Quantum Criticality, Phys. Rev. Lett. 116, 070407 (2016).

[27] M. Fitzpatrick, N. M. Sundaresan, A. C. Y. Li, J. Koch, and A. A. Houck, Observation of a Dissipative Phase Transition in a One-Dimensional Circuit QED Lattice, Phys. Rev. X 7 , 011016 (2017).

[28] T. Gao, E. Estrecho, K. Y. Bliokh, T. C. H. Liew, M. D. Fraser, S. Brodbeck, M. Kamp, C. Schneider, S. Höfling, Y. Yamamoto, F. Nori, Y. S. Kivshar, A. G. Truscott, R. G. Dall, and E. A. Ostrovskaya, Observation of Non-Hermitian Degeneracies in a Chaotic Exciton-Polariton Billiard, Nature (London) 526, 554 (2015).

[29] V. Fernández-Hurtado, J. Mur-Petit, J. J. García-Ripoll, and R. A. Molina, Lattice Scars: Surviving in an Open Discrete Billiard, New J. Phys. 16, 035005 (2014).

[30] F. Iemini, A. Russomanno, J. Keeling, M. Schirò, M. Dalmonte, and R. Fazio, Boundary Time Crystals, Phys. Rev. Lett. 121, 035301 (2018).

[31] K. Tucker, B. Zhu, R. J. Lewis-Swan, J. Marino, F. Jimenez, J. G. Restrepo, and A. M. Rey, Shattered Time: Can a Dissipative Time Crystal Survive Many-Body Correlations?, New J. Phys. 20, 123003 (2018).

[32] B. Zhu, J. Marino, N. Y. Yao, M. D. Lukin, and E. A. Demler, Dicke Time Crystals in Driven-Dissipative Quantum Many-Body Systems, New J. Phys. 21, 073028 (2019).

[33] H. Weimer, A. Kshetrimayum, and R. Orús, Simulation Methods for Open Quantum Many-Body Systems, arXiv: 1907.07079.

[34] M. B. Plenio and P. L. Knight, The Quantum-Jump Approach to Dissipative Dynamics in Quantum Optics, Rev. Mod. Phys. 70, 101 (1998).

[35] J. Dalibard, Y. Castin, and K. Mølmer, Wave-Function Approach to Dissipative Processes in Quantum Optics, Phys. Rev. Lett. 68, 580 (1992).

[36] L. Tian and H. J. Carmichael, Quantum Trajectory Simulations of Two-State Behavior in an Optical Cavity Containing One Atom, Phys. Rev. A 46, R6801 (1992).

[37] J. Jin, D. Rossini, R. Fazio, M. Leib, and M. J. Hartmann, Photon Solid Phases in Driven Arrays of Nonlinearly Coupled Cavities, Phys. Rev. Lett. 110, 163605 (2013).

[38] J. Jin, D. Rossini, M. Leib, M. J. Hartmann, and R. Fazio, Steady-State Phase Diagram of a Driven QED-Cavity Array with Cross-Kerr Nonlinearities, Phys. Rev. A 90, 023827 (2014).

[39] T. E. Lee, S. Gopalakrishnan, and M. D. Lukin, Unconventional Magnetism via Optical Pumping of Interacting Spin Systems, Phys. Rev. Lett. 110, 257204 (2013).

[40] A. Le Boité, G. Orso, and C. Ciuti, Steady-State Phases and Tunneling-Induced Instabilities in the Driven Dissipative Bose-Hubbard Model, Phys. Rev. Lett. 110, 233601 (2013).

[41] A. Le Boité, G. Orso, and C. Ciuti, Bose-Hubbard Model: Relation between Driven-Dissipative Steady States and Equilibrium Quantum Phases, Phys. Rev. A 90, 063821 (2014).

[42] A. Tomadin, V. Giovannetti, R. Fazio, D. Gerace, I. Carusotto, H. E. Türeci, and A. Imamoglu, Signatures of the Superfluid-Insulator Phase Transition in Laser-Driven 
Dissipative Nonlinear Cavity Arrays, Phys. Rev. A 81, 061801(R) (2010).

[43] S. Diehl, A. Tomadin, A. Micheli, R. Fazio, and P. Zoller, Dynamical Phase Transitions and Instabilities in Open Atomic Many-Body Systems, Phys. Rev. Lett. 105, 015702 (2010).

[44] E. P. Wigner, On the Quantum Correction for Thermodynamic Equilibrium, in Part I: Physical Chemistry. Part II: Solid State Physics, edited by A. S. Wightman (Springer, Berlin, Heidelberg, 1997), pp. 110-120.

[45] P. D. Drummond and C. W. Gardiner, Generalised PRepresentations in Quantum Optics, J. Phys. A 13, 2353 (1980).

[46] K.E. Cahill and R.J. Glauber, Density Operators and Quasiprobability Distributions, Phys. Rev. 177, 1882 (1969).

[47] A. Gilchrist, C. W. Gardiner, and P. D. Drummond, Positive P Representation: Application and Validity, Phys. Rev. A 55, 3014 (1997).

[48] P. Deuar, A. Ferrier, M. Matuszewski, G. Orso, and M. H. Szymańska, Fully Quantum Scalable Description of Driven-Dissipative Lattice Models, PRX Quantum 2, 010319 (2021).

[49] J. Schachenmayer, A. Pikovski, and A. M. Rey, ManyBody Quantum Spin Dynamics with Monte Carlo Trajectories on a Discrete Phase Space, Phys. Rev. X 5, 011022 (2015).

[50] A. Biella, J. Jin, O. Viyuela, C. Ciuti, R. Fazio, and D. Rossini, Linked Cluster Expansions for Open Quantum Systems on a Lattice, Phys. Rev. B 97, 035103 (2018).

[51] H. Weimer, Variational Principle for Steady States of Dissipative Quantum Many-Body Systems, Phys. Rev. Lett. 114, 040402 (2015).

[52] H. Weimer, Variational Analysis of Driven-Dissipative Rydberg Gases, Phys. Rev. A 91, 063401 (2015).

[53] M. J. Hartmann and G. Carleo, Neural-Network Approach to Dissipative Quantum Many-Body Dynamics, Phys. Rev. Lett. 122, 250502 (2019).

[54] F. Vicentini, A. Biella, N. Regnault, and C. Ciuti, Variational Neural-Network Ansatz for Steady States in Open Quantum Systems, Phys. Rev. Lett. 122, 250503 (2019).

[55] N. Yoshioka and R. Hamazaki, Constructing Neural Stationary States for Open Quantum Many-Body Systems, Phys. Rev. B 99, 214306 (2019).

[56] S. Finazzi, A. Le Boité, F. Storme, A. Baksic, and C. Ciuti, Corner-Space Renormalization Method for Driven-Dissipative Two-Dimensional Correlated Systems, Phys. Rev. Lett. 115, 080604 (2015).

[57] R. Ors, A Practical Introduction to Tensor Networks: Matrix Product States and Projected Entangled Pair States, Ann. Phys. (Amsterdam) 349, 117 (2014).

[58] M. B. Hastings, An Area Law for One-Dimensional Quantum Systems, J. Stat. Mech. (2007) P08024.

[59] F. G. S. L. Brando, T. S. Cubitt, A. Lucia, S. Michalakis, and D. Perez-Garcia, Area Law for Fixed Points of Rapidly Mixing Dissipative Quantum Systems, J. Math. Phys. (N.Y.) 56, 102202 (2015).

[60] M. J. Kastoryano and J. Eisert, Rapid Mixing Implies Exponential Decay of Correlations, J. Math. Phys. (N.Y.) 54, 102201 (2013).
[61] T. S. Cubitt, A. Lucia, S. Michalakis, and D. Perez-Garcia, Stability of Local Quantum Dissipative Systems, Commun. Math. Phys. 337, 1275 (2015).

[62] A. H. Werner, D. Jaschke, P. Silvi, M. Kliesch, T. Calarco, J. Eisert, and S. Montangero, Positive Tensor Network Approach for Simulating Open Quantum Many-Body Systems, Phys. Rev. Lett. 116, 237201 (2016).

[63] J. Cui, J. I. Cirac, and M. C. Bañuls, Variational Matrix Product Operators for the Steady State of Dissipative Quantum Systems, Phys. Rev. Lett. 114, 220601 (2015).

[64] E. Mascarenhas, H. Flayac, and V. Savona, Matrix-ProductOperator Approach to the Nonequilibrium Steady State of Driven-Dissipative Quantum Arrays, Phys. Rev. A 92, 022116 (2015).

[65] F. Verstraete, J. J. García-Ripoll, and J. I. Cirac, Matrix Product Density Operators: Simulation of Finite-Temperature and Dissipative Systems, Phys. Rev. Lett. 93, 207204 (2004).

[66] A. A. Gangat, T. I, and Y.-J. Kao, Steady States of InfiniteSize Dissipative Quantum Chains via Imaginary Time Evolution, Phys. Rev. Lett. 119, 010501 (2017).

[67] A. Kshetrimayum, H. Weimer, and R. Orús, A Simple Tensor Network Algorithm for Two-Dimensional Steady States, Nat. Commun. 8, 1291 (2017).

[68] D. Kilda, A. Biella, M. Schiró, R. Fazio, and J. Keeling, On the Stability of the Infinite Projected Entangled Pair Operator Ansatz for Driven-Dissipative 2D Lattices, arXiv: 2012.03095.

[69] P. Czarnik, J. Dziarmaga, and P. Corboz, Time Evolution of an Infinite Projected Entangled Pair State: An Efficient Algorithm, Phys. Rev. B 99, 035115 (2019).

[70] P. Czarnik and J. Dziarmaga, Projected Entangled Pair States at Finite Temperature: Iterative Self-Consistent Bond Renormalization for Exact Imaginary Time Evolution, Phys. Rev. B 92, 035120 (2015).

[71] H. N. Phien, J. A. Bengua, H. D. Tuan, P. Corboz, and R. Orús, Infinite Projected Entangled Pair States Algorithm Improved: Fast Full Update and Gauge Fixing, Phys. Rev. B 92, 035142 (2015).

[72] G. Evenbly, Gauge Fixing, Canonical Forms, and Optimal Truncations in Tensor Networks with Closed Loops, Phys. Rev. B 98, 085155 (2018).

[73] M. Kliesch, D. Gross, and J. Eisert, Matrix-Product Operators and States: NP-Hardness and Undecidability, Phys. Rev. Lett. 113, 160503 (2014).

[74] R. J. Baxter, Dimers on a Rectangular Lattice, J. Math. Phys. (N.Y.) 9, 650 (1968).

[75] R. J. Baxter, Variational Approximations for Square Lattice Models in Statistical Mechanics, J. Stat. Phys. 19, 461 (1978).

[76] T. Nishino and K. Okunishi, Corner Transfer Matrix Renormalization Group Method, J. Phys. Soc. Jpn. 65, 891 (1996).

[77] T. Nishino and K. Okunishi, Corner Transfer Matrix Algorithm for Classical Renormalization Group, J. Phys. Soc. Jpn. 66, 3040 (1997).

[78] R. Orús and G. Vidal, Simulation of Two-Dimensional Quantum Systems on an Infinite Lattice Revisited: Corner Transfer Matrix for Tensor Contraction, Phys. Rev. B 80, 094403 (2009). 
[79] P. Corboz, J. Jordan, and G. Vidal, Simulation of Fermionic Lattice Models in Two Dimensions with Projected Entangled-Pair States: Next-Nearest Neighbor Hamiltonians, Phys. Rev. B 82, 245119 (2010).

[80] M. T. Fishman, L. Vanderstraeten, V. Zauner-Stauber, J. Haegeman, and F. Verstraete, Faster Methods for Contracting Infinite Two-Dimensional Tensor Networks, Phys. Rev. B 98, 235148 (2018).

[81] An extension of the hybrid method presented in Ref. [66] to two dimensions appears straightforward; however, increasing the local Hilbert space dimension of the iPEPO to accommodate, for example, an 8-local nearest-neighbor operator would introduce significant computational cost.

[82] X. Wang, C.-S. Yu, and X. Yi, An Alternative Quantum Fidelity for Mixed States of Qudits, Phys. Lett. A 373, 58 (2008).

[83] M. Foss-Feig, J. T. Young, V. V. Albert, A. V. Gorshkov, and M. F. Maghrebi, Solvable Family of Driven-Dissipative Many-Body Systems, Phys. Rev. Lett. 119, 190402 (2017).

[84] I. Carusotto and C. Ciuti, Quantum Fluids of Light, Rev. Mod. Phys. 85, 299 (2013).

[85] T. E. Lee, S. Gopalakrishnan, and M. D. Lukin, Unconventional Magnetism via Optical Pumping of Interacting Spin Systems, Phys. Rev. Lett. 110, 257204 (2013).

[86] M. F. Maghrebi and A. V. Gorshkov, Nonequilibrium ManyBody Steady States via Keldysh Formalism, Phys. Rev. B 93, 014307 (2016).

[87] R. Orús and G. Vidal, Infinite Time-Evolving Block Decimation Algorithm Beyond Unitary Evolution, Phys. Rev. B 78, 155117 (2008).

[88] G. Vidal, Efficient Classical Simulation of Slightly Entangled Quantum Computations, Phys. Rev. Lett. 91, 147902 (2003).
[89] V. Zauner-Stauber, L. Vanderstraeten, M. T. Fishman, F. Verstraete, and J. Haegeman, Variational Optimization Algorithms for Uniform Matrix Product States, Phys. Rev. B 97, 045145 (2018).

[90] J. Haegeman, C. Lubich, I. Oseledets, B. Vandereycken, and F. Verstraete, Unifying Time Evolution and Optimization with Matrix Product States, Phys. Rev. B 94, 165116 (2016).

[91] L. Vanderstraeten, J. Haegeman, and F. Verstraete, TangentSpace Methods for Uniform Matrix Product States, SciPost Phys. Lect. Notes 7 (2019).

[92] A. Nietner, B. Vanhecke, F. Verstraete, J. Eisert, and L. Vanderstraeten, Efficient Variational Contraction of TwoDimensional Tensor Networks with a Non-trivial Unit Cell, Quantum 4, 328 (2020).

[93] N. Lambert, Y.-N. Chen, Y.-C. Cheng, C.-M. Li, G.-Y. Chen, and F. Nori, Quantum Biology, Nat. Phys. 9, 10 (2013).

[94] G. D. Scholes, G. R. Fleming, L. X. Chen, A. AspuruGuzik, A. Buchleitner, D. F. Coker, G. S. Engel, R. van Grondelle, A. Ishizaki, D. M. Jonas, J. S. Lundeen, J. K. McCusker, S. Mukamel, J. P. Ogilvie, A. Olaya-Castro, M. A. Ratner, F. C. Spano, K. B. Whaley, and X. Zhu, Using Coherence to Enhance Function in Chemical and Biophysical Systems, Nature (London) 543, 647 (2017).

[95] R. Penrose, A Generalized Inverse for Matrices, in Mathematical Proceedings of the Cambridge Philosophical Society (Cambridge University Press, Cambridge, England, 1955), Vol. 51, pp. 406-413.

[96] S. Krmer, D. Plankensteiner, L. Ostermann, and H. Ritsch, QuantumOptics.jl: A Julia Framework for Simulating Open Quantum Systems, Comput. Phys. Commun. 227, 109 (2018). 